TRANSACTIONS OF THE

AMERICAN MATHEMATICAL SOCIETY

Volume 355, Number 3, Pages 1043-1066

S 0002-9947(02)03106-9

Article electronically published on October 24, 2002

\title{
NOETHERIAN PI HOPF ALGEBRAS ARE GORENSTEIN
}

\author{
Q.-S. WU AND J. J. ZHANG
}

ABstract. We prove that every noetherian affine PI Hopf algebra has finite injective dimension, which answers a question of Brown (1998).

\section{Introduction}

A classical result of Cartier states that every affine (more generally, locally algebraic) group scheme over a field of characteristic 0 is smooth [DG II.§6.1.1]. Since there is a canonical anti-equivalence between the category of affine group schemes and the category of affine commutative Hopf algebras DG. II.§1.1.6], every affine commutative Hopf algebra over a field of characteristic 0 is regular. An algebra is said to be regular (respectively, Gorenstein) if it has finite global dimension (respectively, finite injective dimension). In the case of positive characteristic, there are non-regular affine commutative Hopf algebras, but every affine commutative Hopf algebra is Gorenstein [Br, 2.3].

In the noncommutative case the geometric machinery becomes invalid and the structure of Hopf algebras seems more difficult to reveal. Some naive extensions of the commutative results do not hold in the noncommutative case. For example, over any field, there are finite dimensional Hopf algebras that are not regular. However, there are several surprising results in the finite dimensional case. Larson and Sweedler proved that every finite dimensional Hopf algebra is Frobenius, namely, Gorenstein of injective dimension $0[\mathrm{LS}]$. Larson and Radford proved that if $H$ is a finite dimensional Hopf algebra over a field of characteristic 0, then the following are equivalent: (a) $H$ is semisimple, namely, $H$ is regular of global dimension 0 , (b) $H$ is cosemisimple, and (c) its antipode is involutory, namely, $S^{2}=\mathrm{id}_{H}$ [LR1], LR2.

During the last fifteen years, as quantum groups or "the function rings of quantum groups" have been studied extensively, more and more people have become interested in infinite dimensional Hopf algebras. A few years ago Brown and Goodearl started to study ring-theoretic properties of a class of infinite dimensional Hopf algebras $[\mathrm{Br}],[\mathrm{BG}]$. They verified that many examples of noetherian affine PI Hopf algebras, some of which are quantum groups at roots of unity, are Gorenstein, and

Received by the editors May 22, 2002.

2000 Mathematics Subject Classification. Primary 16E10, 16W30.

Key words and phrases. Hopf algebra, injective dimension.

The first author was supported in part by the NSFC (project 10171016) and the second author was supported in part by the NSF. 
showed that noetherian affine PI, Gorenstein, Hopf algebras have various good homological properties. In [ $\mathrm{Br}]$, Brown posted a list of questions concerning general noetherian PI Hopf algebras, and one of them is the following [Br, Question A]:

Does every noetherian affine PI Hopf algebra have finite left and right injective dimension?

Under the additional hypothesis of "filtered noetherian" we showed that Brown's question has an affirmative answer [WZ1, 0.3]. The proof of [WZ1, 0.3] is based on the dualizing complexes introduced by Yekutieli $\mathrm{Ye}]$.

The first aim of the paper is to answer Brown's question.

Theorem 0.1. If $H$ is a noetherian affine PI Hopf algebra over a field, then it is Gorenstein.

A slightly more general version is given in Theorem 3.5. Note that this generalizes the result of Larson and Sweedler: finite dimensional Hopf algebras are Frobenius [LS].

The second aim is to show that the Gorenstein property is fundamental for other properties. We will use results of Ajitabh-Smith-Zhang AjSZ, Brown [Br], Brown-Goodearl $[\mathrm{BG}$ and Yekutieli-Zhang YZ1 to prove that noetherian PI Hopf algebras have various properties that are useful for understanding the structure of Hopf algebras. Let GKdim denote the Gelfand-Kirillov dimension. Let id denote the injective dimension and let pd denote the projective dimension.

Theorem 0.2. Let $H$ be a noetherian affine PI Hopf algebra of injective dimension $d$.

(1) $H$ is AS-Gorenstein, Auslander-Gorenstein, and Cohen-Macaulay.

(2) $H$ has a quasi-Frobenius artinian ring of fractions.

(3) For every minimal prime $\mathfrak{p}$ of $H$, GKdim $H / \mathfrak{p}=d$. As a consequence, GKdim $H=d$.

(4) $H$ has a resolution of $H$-bimodules

$$
0 \rightarrow H \rightarrow I^{-d} \rightarrow I^{-d+1} \rightarrow \cdots I^{0} \rightarrow 0
$$

such that when restricted to the left-hand side (respectively, the right-hand side), (0.2.1) is a minimal injective resolution of ${ }_{H} H$ (respectively, $H_{H}$ ), and each $I^{-i}$ is pure of GKdim $i$ on the left and the right respectively.

(5) (Auslander-Buchsbaum formula) If $M$ is a noetherian left (or right) $\mathrm{H}$ module of finite projective dimension, then $\operatorname{pd} M+\operatorname{depth} M=d$.

(6) (Bass's theorem) If $M$ is a noetherian left (or right) $H$-module of finite injective dimension, then id $M=d$.

Some definitions are given in Section 3. Note that a version of Theorem 0.2 holds for commutative Gorenstein local rings. By the localization method, it can be extended to a class of commutative Gorenstein non-local rings. In contrast with the commutative case, we cannot use the localization method to prove any of the assertions in Theorem 0.2. It is fortunate that some "global techniques" work for PI Hopf algebras.

One consequence of the Gorenstein property, which we will state separately from Theorem 0.2, is the projectivity of Hopf algebras over Hopf subalgebras. Nichols and Zoeller proved the following Hopf freeness theorem: a finite dimensional Hopf algebra is a free module over any sub-Hopf algebra [NZ]. Hopf freeness was studied by other authors for infinite dimensional Hopf algebras (see [NR], $\mathrm{Ra}$, Ta1], [Ta2]). 
We consider a slightly more general situation, namely, the projectivity criterion of $H^{\prime}$ over $H$ when there is an algebra homomorphism $H \rightarrow H^{\prime}$ between two Hopf algebras.

Theorem 0.3. Let $H$ and $H^{\prime}$ be noetherian affine PI Hopf algebras of the same injective dimension. Suppose that there is an algebra homomorphism $H \rightarrow H^{\prime}$ such that $H^{\prime}$ is a noetherian $H$-module on both sides. If either id $H_{H}^{\prime}<\infty$ or $\operatorname{pd}_{H} H^{\prime}<\infty$ (e.g. $H$ is regular), then both $H_{H}^{\prime}$ and ${ }_{H} H^{\prime}$ are projective.

Theorem 0.3 plays a key role in the proof of the following result: Let $H$ be an involutory Hopf algebra over a field of characteristic 0 . If $H$ is a finitely generated module over its affine center, then $H$ is regular [WZ3, 0.1]. How to recognize when an infinite dimensional noetherian Hopf algebra is regular is another question asked by Brown $[\mathrm{Br}$, Question $\mathrm{C}]$, which does not yet have a complete answer.

Some basic lemmas about PI algebras are collected in Section 1, and an inductive step of the proof of the main result is described in Section 2. The proofs of Theorems 0.1 and 0.2 are given in Section 3 and the proof of Theorem 0.3 is given in Section 4. Some slightly different versions of these results are discussed in Section 5 .

\section{Preliminaries on PI algebras}

Let $k$ be a base field of arbitrary characteristic. A bimodule or an algebra is said to be noetherian if it is noetherian on both sides. An algebra is called affine if it is finitely generated over the base field. Let $A^{\circ}$ denote the opposite ring of $A$. We usually work with left modules and we adopt the convention of identifying $A^{\circ}$-modules with right $A$-modules. A polynomial identity algebra, or PI algebra for short, is an algebra satisfying a polynomial identity. We refer to [MR, Ch. 13] for some basic materials about PI algebras.

We refer to [MR, 6.8.4] for the definition of dimension function. The basic dimension functions we will use are the Krull dimension, denoted by Kdim, and the Gelfand-Kirillov dimension, denoted by GKdim. Let $\delta$ be a dimension function. We say $\delta$ is exact if $\delta M=\max \{\delta L, \delta N\}$ for every short exact sequence $0 \rightarrow L \rightarrow$ $M \rightarrow N \rightarrow 0$ of finitely generated $A$-modules.

All lemmas in this section are straightforward and well known. We choose to include some of the proofs because ideas in these proofs will be used in later sections. The details of this section could be skipped by readers who are familiar with the material. The following lemma is a collection of results that can be found in [KL].

Lemma 1.1. Let $A$ be a noetherian PI algebra and let $M$ be a noetherian A-module.

(1) If GKdim $M$ is finite, then it is an integer.

(2) GKdim $A=\operatorname{GKdim} A / \mathfrak{p}$ for some prime ideal $\mathfrak{p}$.

(3) GKdim is exact.

(4) GKdim is symmetric in the following sense: if $A$ and $B$ are two noetherian $P I$ algebras and if $M$ is a noetherian $A$-B-bimodule, then GKdim ${ }_{A} M=$ GKdim $M_{B}$.

(5) $\operatorname{GKdim} M \geq \operatorname{Kdim} M$.

Lemma 1.2. Let $A$ be a noetherian PI algebra and let $M$ be a noetherian A-module.

(1) Kdim is exact. 
(2) Kdim is symmetric in the following sense: if $A$ and $B$ are two noetherian $P I$ algebras and if $M$ is a noetherian $A$-B-bimodule, then

$$
\operatorname{Kdim}{ }_{A} M=\operatorname{Kdim} M_{B}=\operatorname{Kdim}\left({ }_{A} M_{B}\right) .
$$

(3) If $A$ is affine, then $\operatorname{Kdim} M=\operatorname{GKdim} M$.

Proof. (1) is [MR, 6.2.4]. (2) follows from [GW, 13.15] and [MR, 6.4.13], and (3) follows from [Row, 6.3.41, 6.3.44].

When we have a noetherian bimodule $M$ as in Lemma 1.2(2), we write $\operatorname{Kdim} M$ for any of $\operatorname{Kdim}_{A} M$, or $\operatorname{Kdim} M_{B}$, or $\operatorname{Kdim}\left({ }_{A} M_{B}\right)$.

Let $A$ be a noetherian algebra. Let $\bmod A$ denote the category of noetherian $A$ modules. Recall that the Krull dimension is defined for all noetherian $A$-modules. For every integer $s$, let $\bmod _{s} A$ be the full subcategory of $\bmod A$ consisting of $A$ modules of $\operatorname{Kdim} \leq s$. Hence $\bmod _{s} A$ is a dense subcategory of $\bmod A$ and we may form a quotient category $\bmod A / \bmod _{s} A$. The basic properties of a quotient category can be found in [Po, Ch.4].

Let $\operatorname{Mod} A$ be the category of all $A$-modules. We can define the Krull dimension of a module to be the maximum of the Krull dimensions of its noetherian submodules. The subcategory $\operatorname{Mod}_{s} A$ and the quotient category $\operatorname{Mod} A / \operatorname{Mod}_{s} A$ can be defined similarly.

For every $A$-module $M$, we denote by $\tau_{s}(M)$ the largest submodule of $M$ of $\operatorname{Kdim} \leq s$. So we have a filtration of submodules

$$
\tau_{0}(M) \subset \tau_{1}(M) \subset \cdots \subset M
$$

If $M$ is an $A$-module of $\operatorname{Kdim} s$, we define the $s$-rank of $M$, denoted by $\operatorname{rank}_{s}(M)$, to be the length of $M$ as an object in the quotient category $\operatorname{Mod} A / \operatorname{Mod}_{s-1} A$. If $M$ is a noetherian $A$-module of $\operatorname{Kdim} s$, then the image of $M$ in $\operatorname{Mod} A / \operatorname{Mod}_{s-1} A$ (also in $\bmod A / \bmod _{s-1} A$ ) is artinian, and hence $\operatorname{rank}_{s}(M)$ is finite.

Let $M$ and $N$ be two $A$-modules. An $A$-module homomorphism $f: M \rightarrow N$ is called an isomorphism modulo Kdim $s$ if the corresponding map in $\operatorname{Mod} A / \operatorname{Mod}_{s} A$ is an isomorphism. In other words, $f$ is an isomorphism modulo Kdim $s$ if and only if the kernel and the cokernel of $f$ have Kdim less than or equal to $s$. It is easy to check that the composition of two isomorphisms modulo Kdim $s$ is again an isomorphism modulo $\mathrm{Kdim} s$. The definitions of surjective and injective maps modulo Kdim $s$ are similar. A complex of $A$-modules

$$
\cdots \rightarrow M^{i-1} \rightarrow M^{i} \rightarrow M^{i+1} \rightarrow \cdots
$$

is said to be exact modulo Kdim $s$ if the corresponding complex in the level of the quotient category $\operatorname{Mod} A / \operatorname{Mod}_{s} A$ is exact, or if, equivalently, every cohomology of the complex as an $A$-module has $\mathrm{Kdim} \leq s$. The following lemma is [Po, 4.3.10].

Lemma 1.3. Suppose $0 \rightarrow L \rightarrow M \stackrel{f}{\rightarrow} N \rightarrow 0$ is a complex of $A$-modules that is exact modulo Kdim $s$. Then there is a short exact sequence of $A$-modules $0 \rightarrow L^{\prime} \rightarrow$ $M^{\prime} \rightarrow N^{\prime} \rightarrow 0$ such that the following hold.

(1) All $L^{\prime}, M^{\prime}$ and $N^{\prime}$ have no nonzero submodule of Kdim $\leq s$. 
(2) The following is a commutative diagram of A-modules:

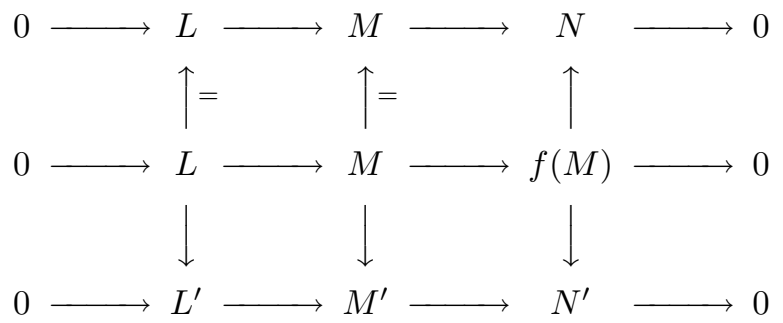

(3) All vertical maps in part (2) are isomorphisms modulo Kdim $s$.

(4) If $M$ is a noetherian $A$-module, then $L^{\prime}, M^{\prime}$ and $N^{\prime}$ can be chosen to be noetherian.

Proof. Let $M^{\prime}=M / \tau_{s}(M)$. Then $M \rightarrow M^{\prime}$ is an isomorphism modulo Kdim $s$. There is a commutative diagram

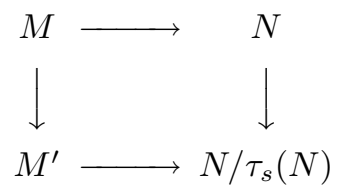

Let $N^{\prime}$ be the image of $M \rightarrow N \rightarrow N / \tau_{s}(N)$, which is also the image of $M^{\prime} \rightarrow$ $N / \tau_{s}(N)$, and let $L^{\prime}$ be the kernel of the surjective map $M^{\prime} \rightarrow N^{\prime}$. The assertions are easy to check now.

Let $M$ be an $A$-module. The left annihilator of $M$ in $A$ is denoted by $\ln \operatorname{ann}_{A}(M)$.

Lemma 1.4. Let $A$ and $B$ be noetherian PI algebras. Let $M$ be a noetherian $A$-module. Let $j$ be an integer.

(1) If $M$ is a noetherian $A$-B-bimodule, then $\operatorname{Ext}_{A}^{j}(M, A)$ is a noetherian $B$ A-bimodule.

(2) $\operatorname{Kdim}_{\operatorname{Ext}_{A}^{j}}(M, A)_{A} \leq \operatorname{Kdim}_{A} M$.

(3) If $M \rightarrow M^{\prime}$ is an isomorphism modulo $\operatorname{Kdim} s$, then

$$
\operatorname{Ext}_{A}^{j}\left(M^{\prime}, A\right) \rightarrow \operatorname{Ext}_{A}^{j}(M, A),
$$

viewed as a map of $A^{\circ}$-modules, is an isomorphism modulo $\mathrm{Kdim} s$.

Proof. (1) This follows from an ungraded analogue of [SZ, 3.5].

(2) We use induction on $s=\operatorname{Kdim}_{A} M$.

By the long exact sequence, the exactness of Kdim [Lemma 1.2(1)], and the noetherian property of $M$, it suffices to show that the assertion holds for a nonzero submodule of $M$. Hence we may assume that $M$ is critical and that $\mathfrak{p}:=1 . \operatorname{ann}_{A}(M)$ is a prime ideal of $A$. In this case there is a short exact sequence

$$
0 \rightarrow A / \mathfrak{p} \rightarrow M^{\oplus r} \rightarrow N \rightarrow 0
$$

with $\operatorname{Kdim} N<s$ by an ungraded analogue of [SZ, 2.1(ii)]. By the long exact sequence of $\operatorname{Ext}_{A}^{*}(-, A)$, it suffices to show that the Krull dimension of $\operatorname{Ext}_{A}^{j}(A / \mathfrak{p}, A)$ and $\operatorname{Ext}_{A}^{j}(N, A)$ are no more than $s$. By induction hypothesis, $\operatorname{Kdim}_{\operatorname{Ext}_{A}^{j}}(N, A)<$ $s$. By (1), $\operatorname{Ext}_{A}^{j}(A / \mathfrak{p}, A)$ is noetherian on both sides and we can compute the $\mathrm{Kdim}$ on either sides by the symmetry of Kdim [Lemma 1.2(2)]. Since $s=$ $\operatorname{Kdim} M=\operatorname{Kdim} A / \mathfrak{p}$ and $\operatorname{Ext}^{j}(A / \mathfrak{p}, A)$ is a (left) $A / \mathfrak{p}$-module, we obtain that $\operatorname{Kdim} \operatorname{Ext}_{A}^{j}(A / \mathfrak{p}, A) \leq s$. Thus the assertion follows. 
(3) Let $M^{\prime \prime}$ be the image of $M \rightarrow M^{\prime}$. Then we have two short exact sequences

$$
0 \rightarrow L \rightarrow M \rightarrow M^{\prime \prime} \rightarrow 0 \text { and } 0 \rightarrow M^{\prime \prime} \rightarrow M^{\prime} \rightarrow P \rightarrow 0,
$$

where $L$ and $P$ are $A$-modules of $\operatorname{Kdim} \leq s$. By $(2), \operatorname{Ext}_{A}^{i}(L, A)$ has $\operatorname{Kdim} \leq s$ for all $i$. Hence the long exact sequence implies that $\operatorname{Ext}_{A}^{j}\left(M^{\prime \prime}, A\right) \rightarrow \operatorname{Ext}_{A}^{j}(M, A)$ is an isomorphism modulo Kdim $s$. Similarly, $\operatorname{Ext}_{A}^{j}\left(M^{\prime}, A\right) \rightarrow \operatorname{Ext}_{A}^{j}\left(M^{\prime \prime}, A\right)$ is an isomorphism modulo $\mathrm{K} \operatorname{dim} s$. The assertion follows by composing these two maps.

A nonzero $A$-module $M$ is called $s$-pure if $\tau_{s}(M)=M$ and $\tau_{t}(M)=0$ for $t<s$, or equivalently, $s=\operatorname{Kdim} M=\operatorname{Kdim} N$ for all nonzero submodules $N \subset M$. The pure module is the same as the homogeneous module defined in [MR, 6.8.8] for Kdim .

Lemma 1.5. Let $A$ and $B$ be noetherian PI algebras.

(1) If $M$ is a noetherian $A$-B-bimodule, then $\tau_{s}\left({ }_{A} M\right)=\tau_{s}\left(M_{B}\right)$ for all $s$. As a consequence, ${ }_{A} M$ is s-pure if and only if $M_{B}$ is s-pure.

(2) If $M$ is an A-module and $N$ is a noetherian $B$-A-module, then $\operatorname{Kdim} N \otimes_{A}$ $M \leq \operatorname{Kdim} M$.

(3) Let $L \subset M$ be s-pure noetherian A-modules such that $\operatorname{Kdim} M / L<s$. If l. $\operatorname{ann}_{A}(L)=\mathfrak{p}$ is a prime ideal of $A$, then $\mathrm{l}_{\operatorname{ann}}(M)=1 . \operatorname{ann}_{A}(L)=\mathfrak{p}$.

Proof. (1) It is easy to check that $\tau_{s}\left(M_{B}\right)$ is a subbimodule. Since $M$ is noetherian on both sides, $\tau_{s}\left(M_{B}\right)$ is noetherian on both sides. By the symmetry of $\operatorname{Kdim}\left[\right.$ Lemma 1.2(2)], we have $\operatorname{Kdim}_{A}\left(\tau_{s}\left(M_{B}\right)\right)=\operatorname{Kdim}\left(\tau_{s}\left(M_{B}\right)\right)_{B} \leq s$. Hence $\tau_{s}\left(M_{B}\right) \subset \tau_{s}\left({ }_{A} M\right)$. Similarly, we have $\tau_{s}\left({ }_{A} M\right) \subset \tau_{s}\left(M_{B}\right)$. Thus we have proved the first assertion. The second assertion is clear by the first one and the definition.

(2) As in the proof of Lemma 1.4(2), we may assume $M=A / \mathfrak{p}$ for some prime ideal $\mathfrak{p}$ of $A$. It is clear that

$$
\operatorname{Kdim}{ }_{B}\left(N \otimes_{A} M\right)=\operatorname{Kdim}\left(N \otimes_{A} A / \mathfrak{p}\right)_{A} \leq \operatorname{Kdim} A / \mathfrak{p}=\operatorname{Kdim} M .
$$

(3) Tensoring $\mathfrak{p} \rightarrow A$ with $0 \rightarrow L \rightarrow M \rightarrow M / L \rightarrow 0$, we obtain

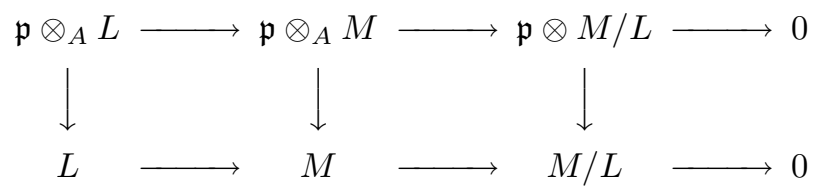

The image of the first vertical map is zero by the definition of $\mathfrak{p}$. By (2), we have $\operatorname{Kdim}\left(\mathfrak{p} \otimes_{A} M / L\right)<s$. Hence the image of the middle vertical map has $\operatorname{Kdim}<s$. Since $M$ is $s$-pure, the image is zero and $\mathfrak{p}=1 . \operatorname{ann}_{A}(M)$.

Lemma 1.5(3) will be used implicitly several times. For example, Lemma 1.5(3) implies that if $M$ is a noetherian $A$ - $B$-bimodule which is critical as a bimodule, then l. $\operatorname{ann}_{A}(M)$ is a prime ideal of $A$ and r. $\operatorname{ann}_{B}(M)$ is a prime ideal of $B$. From now on, when we have a noetherian bimodule $M$ we will just use $\tau_{s}(M)$ for either $\tau_{s}\left({ }_{A} M\right)$ or $\tau_{s}\left(M_{B}\right)$. For our convenience in the next section, we state the next two lemmas for a ring $B$ instead of $A$. The next lemma is a special case of [MR, 6.8.16].

Lemma 1.6. Let $B$ be a noetherian PI algebra. If $B_{B}$ and ${ }_{B} B$ are pure, then $B$ has an artinian ring of fractions. 
Let $\mathcal{C}_{B}(0)$ denote the set of regular elements of $B$. If $I$ is an ideal of $B$, let $\mathcal{C}_{B}(I) \subset B$ be the pre-image of $\mathcal{C}_{B / I}(0)$. If $B$ has an artinian ring of fractions (e.g., in Lemma 1.6), then by Small's theorem [MR 4.1.4], $\mathcal{C}_{B}(0)=\mathcal{C}_{B}(N)$, where $N$ is the prime radical of $B$. It is clear that $\mathcal{C}_{B}(N)=\bigcap_{j} \mathcal{C}_{B}\left(\mathfrak{p}_{j}\right)$, where $\left\{\mathfrak{p}_{j}\right\}$ is the set of all minimal primes of $B$. For the purpose of this paper, we say that $x \in \mathcal{C}_{B}(0)$ is a special regular element if for every minimal prime $\mathfrak{p}_{i}$ the following hold:

(1) The image $\bar{x} \in B / \mathfrak{p}_{i}$ is in $\left(\bigcap_{j \neq i} \mathfrak{p}_{j}\right) / \mathfrak{p}_{i}$.

(2) $\bar{x}$ is a central regular element in $B / \mathfrak{p}_{i}$.

(3) If $\operatorname{Kdim} B / \mathfrak{p}_{i}>0$, then $\bar{x}$ is not a unit.

Recall that if $D$ is a noetherian prime PI ring, then every noetherian $D^{\circ}$-module $M$ such that $\operatorname{Kdim} M<\operatorname{Kdim} D$ has a nonzero right annihilator r. $\operatorname{ann}_{D}(M)$ GW 8.9]. Since $D$ is prime and PI, there is a central regular element $c$ in $\mathrm{r}$. $\operatorname{ann}_{D}(M)$ such that $M c=0$ [Row, 6.1.28]. If $\operatorname{Kdim} D>0$, we can choose $c$ to be a non-unit.

Lemma 1.7. Let $B$ be a noetherian PI algebra with an artinian ring of fractions. Suppose that $\mathfrak{p}_{t}$ is a minimal prime ideal of $B$ such that $\operatorname{Kdim} B / \mathfrak{p}_{t}=s>0$. Let $L$ be a noetherian $B^{\circ}$-module with $\mathrm{r}$. $\operatorname{ann}_{B}(L)=\mathfrak{p}_{t}$. Then there is a special regular element $x \in \mathcal{C}_{B}(0)$ such that $\operatorname{Kdim} L x / L x^{2}=\operatorname{Kdim} L-1$ and $\tau_{s-1}(L) x=0$.

Proof. For every minimal prime $\mathfrak{p}_{i}$ of $B, \mathfrak{p}_{i}$ does not contain $\bigcap_{j \neq i} \mathfrak{p}_{j}$. Thus there is a central regular element $\overline{c_{i}} \in B / \mathfrak{p}_{i}$ such that (i) a pre-image $c_{i}$ is in $\bigcap_{j \neq i} \mathfrak{p}_{j}$ and (ii) $\overline{c_{i}}$ is not a unit when $\operatorname{Kdim} B / \mathfrak{p}_{i}>0$. Let $x=\sum_{i} c_{i}$. For each $i, \bar{x}=\bar{c}_{i}$ in $B / \mathfrak{p}_{i}$. The properties of $c_{i}$ imply that $x$ is a special regular element.

When $i=t$ we need to choose $c_{i}$ more finely to satisfy other requirements. First we note that $L c_{j}=0$ for all $j \neq t$. Let $D=B / \mathfrak{p}_{t}$. Since $\operatorname{Kdim} \tau_{s-1}(L)<\operatorname{Kdim} D$, there is a central regular element $\overline{c^{\prime}} \in D$ such that $\tau_{s-1}(L) c^{\prime}=0$. Let $M=$ $L / \tau_{s-1}(L)$. Pick a submodule $N \subset M$ such that $\operatorname{Kdim} M / N=s-1$ [GW] 13D and $13 \mathrm{G}]$. Then there is a central regular element $\overline{c^{\prime \prime}} \in D$ such that $(M / N) c^{\prime \prime}=0$. Replace $c_{t}$ by $c_{t} c^{\prime} c^{\prime \prime}$ and let $x$ be constructed as in the previous paragraph by using the new $c_{t}$. Then $\operatorname{Kdim} M / M x=s-1[\mathrm{GW}, 13.7]$ and $\tau_{s-1}(L) x=0$. Note that $L x / L x^{2} \cong M / M x$, and hence $x$ has the required properties.

\section{AN INDUCTIVE STEP}

This section contains a key step of the proof of Theorem 0.1. The material presented here is technical. Throughout this section we assume $A$ and $B$ are noetherian PI algebras.

Hypothesis 2.1. Suppose there is a subset $\Phi \subset \mathbb{Z}$ such that the following hold.

(1) If $i \in \Phi$, then $\operatorname{Ext}_{A}^{i}(S, A) \neq 0$ for all simple $A$-modules $S$, and $\operatorname{Ext}_{A}^{i}(-, A)$ is an exact functor on $A$-modules of finite length.

(2) If $i \notin \Phi$, then $\operatorname{Ext}_{A}^{i}(S, A)=0$ for all simple $A$-modules $S$.

We will see in the next section that noetherian affine PI Hopf algebras satisfy Hypothesis 2.1. Starting from Hypothesis 2.1, we want to prove a higher dimensional version of it. To do so, we need to add a right $B$-module structure to carry on the inductive step.

Hypothesis 2.2. Let $s$ be an integer.

(1s). For every $i, \operatorname{Ext}_{A}^{i}(-, A)$ is an exact functor modulo $\operatorname{Kdim}(s-1)$ when applied to noetherian $A$ - $B$-modules of $\operatorname{Kdim} \leq s$. 
(2s). For every $i \in \Phi$ (where $\Phi$ is given in Hypothesis 2.1), $\operatorname{Kdim}_{\operatorname{Ext}}^{i-s}(M, A)$ $=s$ for all noetherian $A$-B-modules $M$ of $\operatorname{Kdim} s$.

When $s=0$, Hypothesis 2.2 follows from Hypothesis 2.1. We need a few lemmas to show that Hypothesis $2.2(1 \mathrm{~s}, 2 \mathrm{~s})$ follows from Hypothesis 2.1 for all $s$.

Lemma 2.3. Let $i$ be a fixed integer.

(1) If $\operatorname{Ext}_{A}^{i}(S, A)=0$ for all simple $A$-modules $S$, then $\operatorname{Kdim}_{\operatorname{Ext}}{ }_{A}^{i-s}(M, A)<s$ for all noetherian $A$-modules $M$ of $\mathrm{Kdim} \leq s$.

(2) If $\operatorname{Ext}_{A}^{j}(S, A)=0$ for all $i-s \leq j \leq i$ and all simple $A$-modules $S$, then $\operatorname{Ext}_{A}^{i-s}(M, A)=0$ for all noetherian $A$-modules $M$ of $\mathrm{Kdim} \leq s$.

(3) If $\operatorname{Ext}_{A}^{j}(S, A)=0$ for all $j \leq i$ and all simple $A$-modules $S$, then $\operatorname{Ext}_{A}^{j-s}(M$, $A)=0$ for all $j \leq i$ and all noetherian $A$-modules $M$ such that $\operatorname{Kdim} M \leq$ $s$.

(4) If $\operatorname{Ext}_{A}^{j}(S, A)=0$ for all $j \geq i$ and all simple $A$-modules $S$, then $\operatorname{Ext}_{A}^{j}(M$, $A)=0$ for all $j \geq i$ and all noetherian $A$-modules $M$. In particular, id ${ }_{A} A<i$.

(5) Let $M$ be a noetherian $A$-module. If $\operatorname{Tor}_{j}^{A}(S, M)=0$ for all $j \geq i$ and for all simple $A^{\circ}$-modules $S$, then $\operatorname{pd} M<i$.

Proof. (1) We use induction on $s$. If $s=0$, the statement follows from the long exact sequence of $\operatorname{Ext}^{i}(-, A)$ and the noetherian property of $M$. Now we assume the statement holds for $s$. Let $M$ be a noetherian $A$-module with $\operatorname{Kdim} M=s+1$. By the argument in the proof of Lemma 1.4(2), we may assume $M$ is critical and l. $\operatorname{ann}(M)=\mathfrak{p}$ is a prime of $A$. Then there is a short exact sequence (see (1.4.1))

$$
0 \rightarrow A / \mathfrak{p} \rightarrow M^{\oplus r} \rightarrow N \rightarrow 0,
$$

where $N$ is $A / \mathfrak{p}$-torsion. Hence $\operatorname{Kdim} N<s+1$. By Lemma 1.4(2),

$$
\operatorname{Kdim} \operatorname{Ext}_{A}^{j}(N, A) \leq s \quad \text { for all } j .
$$

It follows from the long exact sequence that we only need to show that

$$
\operatorname{Kdim}_{\operatorname{Ext}}^{i-s-1}(A / \mathfrak{p}, A)<s+1 .
$$

Let $B=A / \mathfrak{p}$. Since $\operatorname{Ext}_{A}^{i-s-1}(B, A)$ is a noetherian (left) $B$-module, it has Kdim at

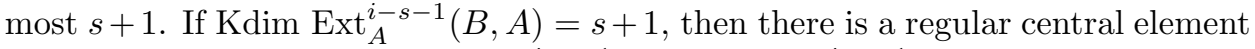
$c \in B$ such that the map $l_{c}: \operatorname{Ext}_{A}^{i-s-1}(B, A) \rightarrow \operatorname{Ext}_{A}^{i-s-1}(B, A)$ has cokernel of $\operatorname{Kdim} s$. Applying $\operatorname{Ext}_{A}^{i}(-, A)$ to the short exact sequence

$$
0 \rightarrow B \stackrel{r_{c}}{\longrightarrow} B \rightarrow N_{c} \rightarrow 0
$$

where $N_{c}=B /(c)$, we obtain an exact sequence

$$
\cdots \rightarrow \operatorname{Ext}_{A}^{i-s-1}(B, A) \stackrel{l_{c}}{\longrightarrow} \operatorname{Ext}_{A}^{i-s-1}(B, A) \rightarrow \operatorname{Ext}_{A}^{i-s}\left(N_{c}, A\right) \rightarrow \cdots .
$$

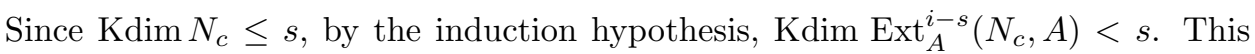
contradicts the fact that the cokernel of $\operatorname{Ext}_{A}^{i-s-1}(B, A) \rightarrow \operatorname{Ext}_{A}^{i-s-1}(B, A)$ has Kdim s. Therefore

$$
\operatorname{Kdim} \operatorname{Ext}_{A}^{i-s-1}(B, A)<s+1 .
$$

(2) We use the induction similar to (1) with some extra care. Assume that $\operatorname{Ext}_{A}^{j}(S, A)=0$ for all $i-s-1 \leq j \leq i$ and for all simple $A$-modules $S$. By 
the induction hypothesis, $\operatorname{Ext}_{A}^{i-s}(N, A)=0$ for all $N$ with $\operatorname{Kdim} N \leq s$. By (1), $\operatorname{Kdim} \operatorname{Ext}_{A}^{i-s-1}(B, A) \leq s$; so there is a regular element $c \in B$ such that

$$
c \operatorname{Ext}_{A}^{i-s-1}(B, A)=0
$$

or in other words $l_{c}=0$. Hence by (2.3.2) the second $\operatorname{Ext}_{A}^{i-s-1}(B, A)$ in $(2.3 .2)$ is a submodule of $\operatorname{Ext}_{A}^{i-s}\left(N_{c}, A\right)$, which is zero.

(3) This is a special case of (2).

(4) This is a special case of [WZ1, 1.4] (note that the $\operatorname{assumption} \operatorname{inj} \operatorname{dim} X<\infty$ is not necessary).

(5) We use induction to show that $\operatorname{Tor}_{j}^{A}(Z, M)=0$ for all $j \geq i$ and all noetherian $A^{\circ}$-modules $Z$. The induction process in the proof of Lemma 1.4(2) implies that we only need to consider $Z=A / \mathfrak{p}$ for some prime $\mathfrak{p}$. If $\operatorname{Kdim} A / \mathfrak{p}=0$, this is clear; so we assume $\operatorname{Kdim} A / \mathfrak{p}>0$.

Let $B=A / \mathfrak{p}$. Consider $T:=\operatorname{Tor}_{j}^{A}(A / \mathfrak{p}, M)$ for some $j \geq i$, which is a noetherian $B$-module. For every regular central element $c$ in $B$, we have a short exact sequence (2.3.1). The corresponding long exact sequence of Tor is

$$
\cdots \rightarrow \operatorname{Tor}_{j}^{A}(B, M) \stackrel{l_{c}}{\longrightarrow} \operatorname{Tor}_{j}^{A}(B, M) \rightarrow \operatorname{Tor}_{j}^{A}\left(N_{c}, M\right) \rightarrow \cdots
$$

By the induction hypothesis, $\operatorname{Tor}_{j}^{A}\left(N_{c}, M\right)=0$. Hence the map $l_{c}$ is always surjective. Let $T_{0}$ be the $B$-torsion submodule of $T$. Then $l_{c}$ becomes bijective on $T / T_{0}$. Thus $T / T_{0} \cong Q(B) \otimes_{B}\left(T / T_{0}\right)$, where $Q(B)$ is the ring of fractions of $B$. But any nonzero $Q(B)$-module is not noetherian over $B$ when $\operatorname{Kdim} B>0$. Hence $T / T_{0}=0$ and $T=T_{0}$. Pick $c$ such that $c T_{0}=0$. Then (2.3.3) shows that the second $\operatorname{Tor}_{j}^{A}(B, M)$ is a submodule of $\operatorname{Tor}_{j}^{A}\left(N_{c}, M\right)$, which is zero.

The above argument shows that the flat dimension of $M$ is less than $i$. Since $M$ and $A$ are noetherian, the projective dimension of $M$ is equal to its flat dimension.

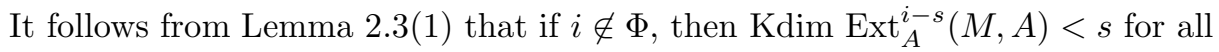
noetherian $A$-modules $M$ with $\operatorname{Kdim} M \leq s$.

Lemma 2.4. Suppose $0 \rightarrow M_{1} \rightarrow M_{2} \rightarrow M_{3} \rightarrow 0$ is a short exact sequence of A-modules. Then the sequence

$$
0 \rightarrow \operatorname{Ext}_{A}^{i}\left(M_{3}, A\right) \rightarrow \operatorname{Ext}_{A}^{i}\left(M_{2}, A\right) \rightarrow \operatorname{Ext}_{A}^{i}\left(M_{1}, A\right) \rightarrow 0
$$

is exact modulo Kdim $s$ for all $i$ if and only if the map

$$
\operatorname{Ext}_{A}^{i}\left(M_{2}, A\right) \rightarrow \operatorname{Ext}_{A}^{i}\left(M_{1}, A\right)
$$

is surjective modulo $\mathrm{Kdim} s$ for all $i$.

Proof. By the long exact sequence, the surjectivity of $\operatorname{Ext}_{A}^{i}\left(M_{2}, A\right) \rightarrow \operatorname{Ext}_{A}^{i}\left(M_{1}, A\right)$ implies the injectivity of $\operatorname{Ext}_{A}^{i+1}\left(M_{3}, A\right) \rightarrow \operatorname{Ext}_{A}^{i+1}\left(M_{2}, A\right)$ for all $i$.

Lemma 2.5. Let $0 \rightarrow L \rightarrow M \rightarrow N \rightarrow 0$ be a short exact sequence of noetherian $(s+1)$-pure $A$-B-bimodules. Suppose that $B$ is $(s+1)$-pure and that $\mathrm{r} \cdot \operatorname{ann}_{B}(L)$ is a minimal prime ideal of $B$. Let $x \in \mathcal{C}_{B}(0)$ be a special regular element.

(1) The sequence

$$
0 \rightarrow L / L x \rightarrow M / M x \rightarrow N / N x \rightarrow 0
$$

is exact. 
(2) Assume Hypothesis 2.2(1s). Then

$$
0 \rightarrow \operatorname{Ext}_{A}^{i}(N / N x, A) \rightarrow \operatorname{Ext}_{A}^{i}(M / M x, A) \rightarrow \operatorname{Ext}_{A}^{i}(L / L x, A) \rightarrow 0
$$

is exact modulo $\operatorname{Kdim}(s-1)$.

Proof. (1) Since $M / M x \cong M \otimes_{B} B / B x$, it suffices to show that the map $L / L x \rightarrow$ $M / M x$ is injective, or to show that $\operatorname{Tor}_{1}^{B}(N, B / B x)=0$. But

$$
\operatorname{Tor}_{1}^{B}(N, B / B x)=\{n \in N \mid n x=0\}=0,
$$

since $N_{B}$ is $(s+1)$-pure.

(2) First of all, since $L$ is $(s+1)$-pure, $x$ is regular on $L$ and $\operatorname{Kdim} L / L x \leq$ $\operatorname{Kdim} L-1=s$. The same holds for $M / M x$ and $N / N x$. By Lemma 2.4, it suffices to show the surjectivity of $\operatorname{Ext}_{A}^{i}(M / M x, A) \rightarrow \operatorname{Ext}_{A}^{i}(L / L x, A)$ for all $i$.

We claim that there is a noetherian $A$-module $\Omega$ such that (i) $\Omega$ contains $M / M x$ as a submodule, and (ii) the map $f: \operatorname{Ext}_{A}^{i}(\Omega, A) \rightarrow \operatorname{Ext}_{A}^{i}(L / L x, A)$ is surjective modulo $\operatorname{Kdim}(s-1)$. If this is true, then the map $g: \operatorname{Ext}_{A}^{i}(M / M x, A) \rightarrow$ $\operatorname{Ext}_{A}^{i}(L / L x, A)$ is surjective modulo $\operatorname{Kdim}(s-1)$, since $f$ factors through $g$.

We now construct $\Omega$.

By Lemma 1.6, $B$ has an artinian ring of fractions, denoted by $Q(B)$. Since $M$ is $(s+1)$-pure, it follows that $M$ is $B^{\circ}$-torsion-free and $M$ is a submodule of $\bar{M}:=M \otimes_{B} Q(B)$. It is clear that the inclusion $M \rightarrow \bar{M}$ is an $A$-B-bimodule map. In the following we show that the $A$-B-subbimodule $M x^{-1} B$ of $\bar{M}$ is noetherian on both sides. Since $M x^{-1} B=M B x^{-1} B$ is a factor of $M \otimes_{B} B x^{-1} B$, it suffices to show that the latter is noetherian on both sides. By the noetherian property of $M$ and induction reduction, we may assume that $M$ is a critical bimodule and $\mathfrak{p}:=\mathrm{r} . \operatorname{ann}_{B}(M)$ is a minimal prime of $B$. Let $c$ be the image of $x$ in $D:=B / \mathfrak{p}$. Then

$$
M \otimes_{B} B x^{-1} B=M \otimes_{D} D c^{-1} D=M c^{-1},
$$

which is isomorphic to $M$ as an $A$-B-bimodule. Hence $M \otimes_{B} B x^{-1} B$ is noetherian on both sides.

Let $\Lambda=M B x^{-1} B$. Then $\Lambda$ is a noetherian $A$-B-subbimodule of $\bar{M}$ that contains $M$. Since $x$ is central in $B / \mathrm{r}$. $\operatorname{ann}_{B}(L), L x^{-1}=L x^{-1} B$ is an $A$-B-subbimodule of $\Lambda$. Hence there is an exact sequence

$$
0 \rightarrow L x^{-1} /\left(L x^{-1} \cap M\right) \rightarrow \Lambda / M \rightarrow P \rightarrow 0
$$

for some $P$. By (1), $M x \cap L=L x$, which is equivalent to $M \cap L x^{-1}=L$. Hence we have an exact sequence of noetherian $A$ - $B$-bimodules

$$
0 \rightarrow L x^{-1} / L \rightarrow \Lambda / M \rightarrow P \rightarrow 0 .
$$

Since $\bar{M} / M$ is right $B$-torsion, $K \operatorname{dim} \Lambda / M \leq s$. By Hypothesis $2.2(1 \mathrm{~s})$, the map $\operatorname{Ext}_{A}^{i}(\Lambda / M, A) \rightarrow \operatorname{Ext}_{A}^{i}\left(L x^{-1} / L, A\right)$ is surjective modulo $\operatorname{Kdim}(s-1)$ for all $i$. Tensoring (2.5.1) with $-\otimes_{B} B x$, we have a short exact sequence

$$
0 \rightarrow L / L x \rightarrow \Lambda x / M x \rightarrow P \otimes_{B} B x \rightarrow 0,
$$

which is isomorphic to (2.5.1) as complexes of $A$-modules. Hence the map

$$
\operatorname{Ext}_{A}^{i}(\Lambda x / M x, A) \rightarrow \operatorname{Ext}_{A}^{i}(L / L x, A)
$$

is surjective modulo $\operatorname{Kdim}(s-1)$ for all $i$. Let $\Omega=\Lambda x / M x$. It is clear that $\Omega$ contains $M / M x$. 
Lemma 2.6. Hypothesis 2.2(1(s+1)) is equivalent to the following:

Hypothesis 2.6(1 $(s+1))$ : for every short exact sequence of noetherian $(s+1)$-pure A-B-bimodules

$$
0 \rightarrow L \rightarrow M \rightarrow N \rightarrow 0
$$

with $\mathrm{r} . \operatorname{ann}_{B}(L)$ being a minimal prime of $B$, the sequence

$$
0 \rightarrow \operatorname{Ext}_{A}^{i}(N, A) \rightarrow \operatorname{Ext}_{A}^{i}(M, A) \rightarrow \operatorname{Ext}_{A}^{i}(L, A) \rightarrow 0
$$

is exact modulo Kdim s for all $i$.

Proof. It is clear that Hypothesis 2.6(1(s+1)) is weaker than 2.2(1(s+1)). We now prove that Hypothesis 2.6(1(s+1)) implies Hypothesis 2.2(1(s+1)). Without loss of generality, we may assume $\mathrm{r} . \operatorname{ann}_{B}(M)=0$.

For every short exact sequence of noetherian $A$ - $B$-bimodules of $\operatorname{Kdim} \leq s+1$,

$$
0 \rightarrow L \rightarrow M \rightarrow N \rightarrow 0
$$

and for every $i$, we need to show that

$$
0 \rightarrow \operatorname{Ext}_{A}^{i}(N, A) \rightarrow \operatorname{Ext}_{A}^{i}(M, A) \rightarrow \operatorname{Ext}_{A}^{i}(L, A) \rightarrow 0
$$

is exact modulo $\operatorname{Kdim} s$. By Lemmas 1.3 and 1.4 we may assume that all modules in (2.6.1) are $(s+1)$-pure. We use induction on the $(s+1)$-rank of the middle term $M$. (The definition of the rank is given in Section 1.) The statement is trivial when the $(s+1)$-rank of $M$ is 1 , since in that case either $L$ or $N$ is zero. Similarly we may assume the $(s+1)$-ranks of $L$ and $N$ are nonzero and strictly less than the $(s+1)$-rank of $M$. Pick a subbimodule $L_{1} \subset L$ such that r. $\operatorname{ann}_{B}\left(L_{1}\right)$ is a minimal prime of $B$ and $M / L_{1}$ is also $(s+1)$-pure (this can be achieved by using Lemma $1.5(3))$. Then we have a diagram of short exact sequences

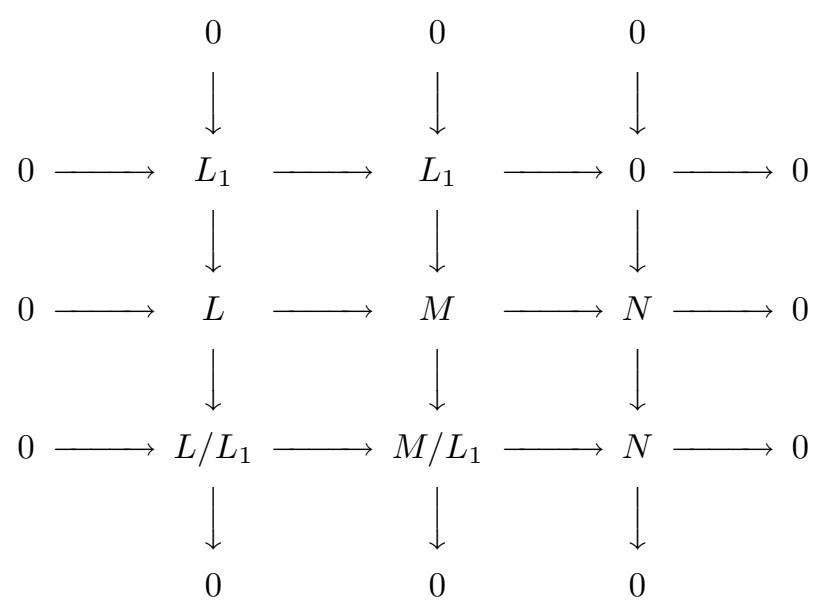


Applying $\operatorname{Ext}_{A}^{i}(-, A)$ to this diagram, we obtain a diagram

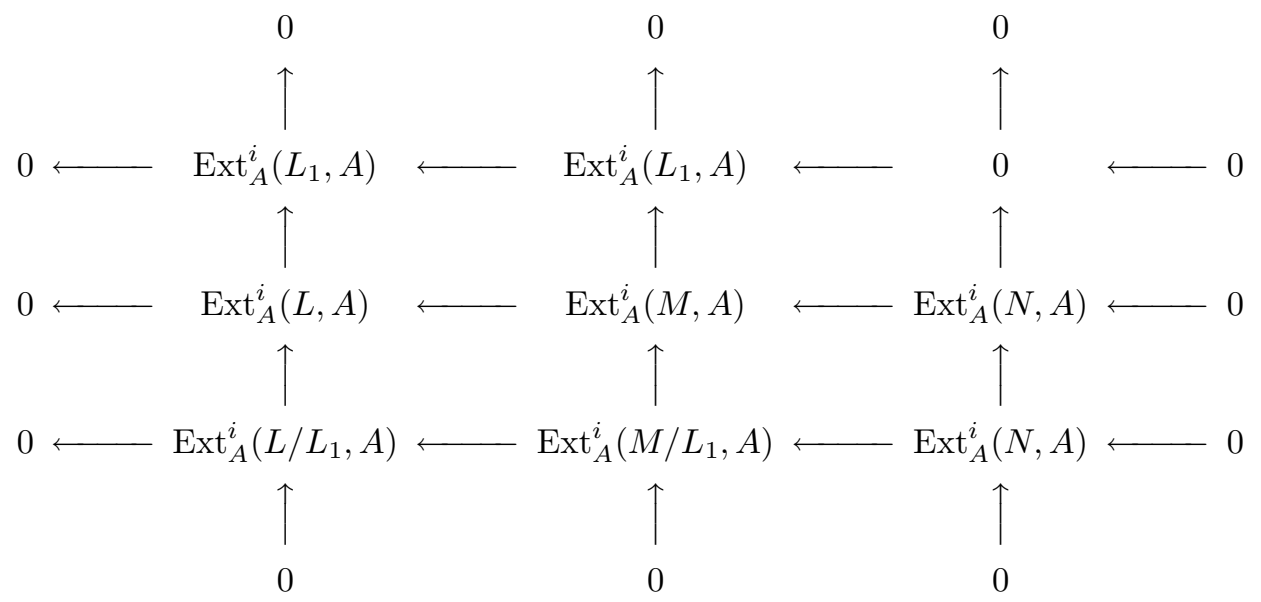

The first column and the third row are exact modulo $\operatorname{Kdim} s$ because the $(s+1)$ rank of $L$ and the $(s+1)$-rank of $M / L_{1}$ are less than the $(s+1)$-rank of $M$. The third column and the first row are trivially exact. The middle column is exact modulo Kdim $s$ because r. $\operatorname{ann}_{B}\left(L_{1}\right)$ is a minimal prime of $B$ and by Hypothesis $2.6(1(\mathrm{~s}+1))$. By a version of the five lemma or an easy computation, the middle row is exact modulo $\mathrm{Kdim} s$.

Lemma 2.7. Assume Hypothesis 2.2(1s). Then Hypothesis 2.2(1(s+1)) holds.

Proof. By Lemma 2.6, it suffices to show Hypothesis 2.6(1(s+1)).

Suppose $0 \rightarrow L \rightarrow M \rightarrow N \rightarrow 0$ is an exact sequence of noetherian $(s+1)$-pure $A$ $B$-bimodules such that $\mathrm{r}$. $\operatorname{ann}_{B}(L)=\mathfrak{p}$ is a minimal prime of $B$. Then $\operatorname{Kdim} B / \mathfrak{p}=$ $s+1>0$. Without loss of generality, we may assume that $\mathrm{r} \cdot \operatorname{ann}_{B}(M)=0$. Since $M$ is $(s+1)$-pure, $B$ is also $(s+1)$-pure by [GW] 8.9] and Lemma 1.5(1).

Since $B$ is $(s+1)$-pure, it has an artinian ring of fractions [Lemma 1.6]. By Lemma 2.4 it suffices to show that the map $\phi: \operatorname{Ext}_{A}^{i-1}(M, A) \rightarrow \operatorname{Ext}_{A}^{i-1}(L, A)$ is surjective modulo Kdim $s$ for all $i$. We prove it by contradiction. Assume that $\phi$ is not surjective modulo Kdim $s$. Let $C$ be the cokernel of $\phi$. Hence $C$ is a noetherian $B / \mathfrak{p}$ - $A$-bimodule of $\operatorname{Kdim}(s+1)$. By Lemma 1.7, there is a special regular element $x \in \mathcal{C}_{B}(0)$ such that $\operatorname{Kdim} x C / x^{2} C=s$ and $x \tau_{s}(C)=0$. In particular, the image $\bar{x}$ is central in $B / \mathfrak{p}$. 
We now consider the diagram

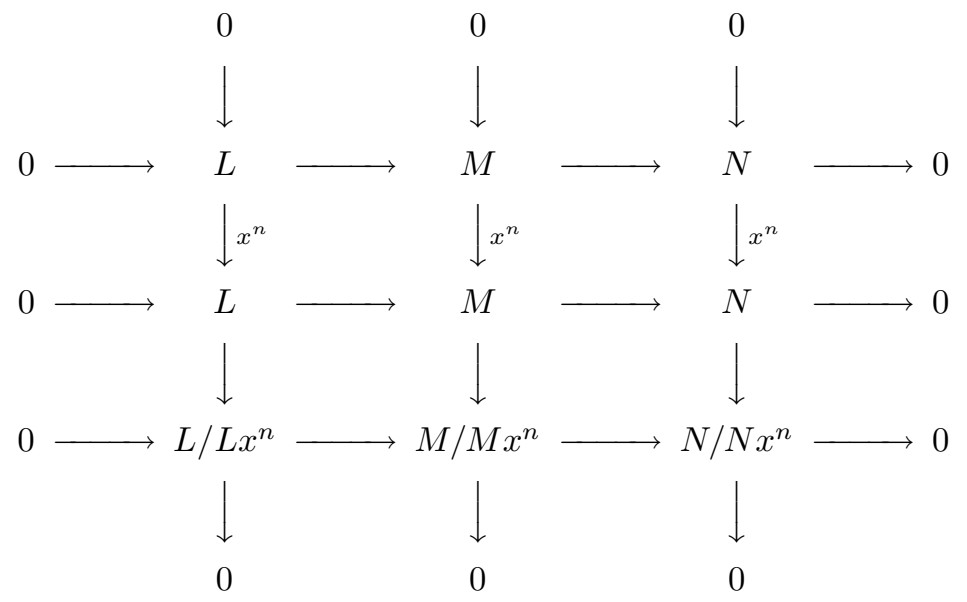

By Lemma 2.5(1) the last row and hence all short exact sequences are exact. For the rest of the proof we write $E^{i}(M)$ for $\operatorname{Ext}_{A}^{i}(M, A)$. Applying $E^{i}(-)$ to the above, we have

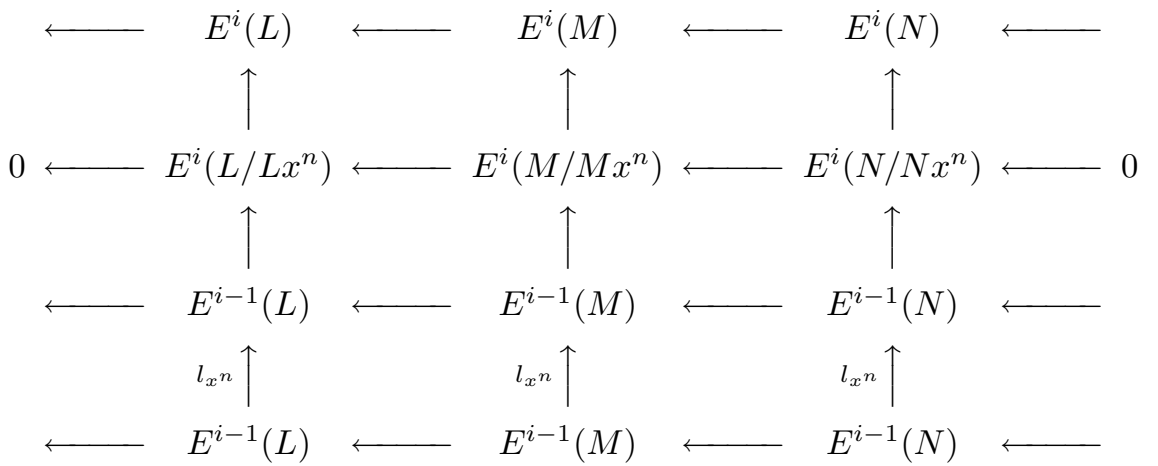

where the second row is exact modulo $\operatorname{Kdim}(s-1)$ by Lemma 2.5(2). Note that $E^{i-1}(M)$ is a $B$-A-bimodule and the left multiplication by $x^{n}$, denoted by $l_{x^{n}}$, is the map given in the above diagram. The cokernel of the middle $l_{x^{n}}$ is mapped into $E^{i}\left(M / M x^{n}\right)$. In other words, the induced map

$$
E^{i-1}(M) / x^{n} E^{i-1}(M) \rightarrow E^{i}\left(M / M x^{n}\right)
$$

is injective. The same holds for $L$ and $N$. We now have a diagram

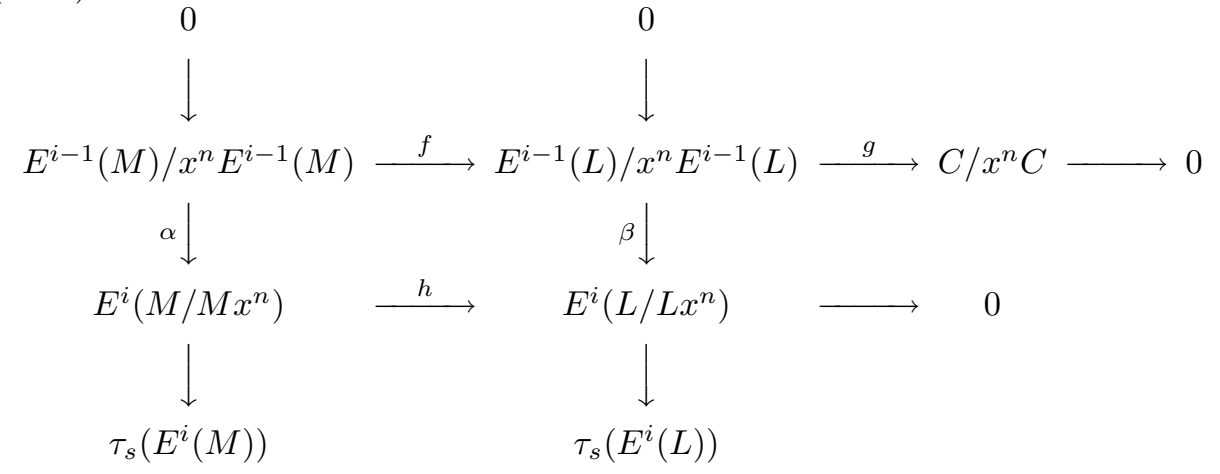


The first row of (2.7.1) is exact since it is obtained by applying $B / x^{n} B \otimes_{B}-$ to an exact sequence. The second row of (2.7.1) is exact modulo $\operatorname{Kdim}(s-1)$ by Lemma $2.5(2)$. The columns are clearly exact. The $s$-ranks of all modules in (2.7.1) are finite, and some of them might be dependent on $n$. The $s$-rank of $\tau_{s}\left(E^{i}(M)\right)$, say $r$, is finite and independent of $n$. Let $K$ be the kernel of $h$. Then the kernel of $f$ is isomorphic to (im $\alpha) \cap K$. Since the $s$-rank is additive with respect to short exact sequences, an easy computation shows that

$$
\begin{aligned}
\operatorname{rank}_{s}\left(C / x^{n} C\right)= & \operatorname{rank}_{s}(\operatorname{ker} f)-\operatorname{rank}_{s}\left(E^{i-1}(M) / x^{n} E^{i-1}(M)\right) \\
& \quad+\operatorname{rank}_{s}\left(E^{i-1}(L) / x^{n} E^{i-1}(L)\right) \\
= & \operatorname{rank}_{s}(\operatorname{ker} f)-\left(\operatorname{rank}_{s}\left(E^{i}\left(M / M x^{n}\right)\right)-\operatorname{rank}_{s}(\operatorname{coker} \alpha)\right) \\
& \left.\quad+\operatorname{rank}_{s}\left(E^{i}\left(L / L x^{n}\right)\right)-\operatorname{rank}_{s}(\operatorname{coker} \beta)\right) \\
= & \operatorname{rank}_{s}(\operatorname{ker} f)-\operatorname{rank}_{s}(\operatorname{ker} h)+\operatorname{rank}_{s}(\operatorname{coker} \alpha)-\operatorname{rank}_{s}(\operatorname{coker} \beta) \\
= & \operatorname{rank}_{s}(\operatorname{im} \alpha \cap K)-\operatorname{rank}_{s}(K)+\operatorname{rank}_{s}(\operatorname{coker} \alpha)-\operatorname{rank}_{s}(\operatorname{coker} \beta) \\
\leq & \operatorname{rank}_{s}(\operatorname{coker} \alpha) \leq r .
\end{aligned}
$$

By the choice of $x$ given in Lemma $1.7, \operatorname{Kdim} x C / x^{2} C=s$ and $x^{n} C / x^{n+1} C \cong$ $x C / x^{2} C$ for all $i$. Thus the $s$-rank of $C / x^{n} C$ cannot be bounded by $r$ for large $n$. This yields a contradiction, as desired.

Lemma 2.8. Suppose Hypothesis 2.2(1s,2s) holds. Let $L$ be a noetherian $A-B$ bimodule with $\operatorname{Kdim} L=s+1$ such that $\mathrm{r} \cdot \operatorname{ann}_{B}(L)$ is a minimal prime of $B$. Then for every $i \in \Phi$,

$$
\operatorname{Kdim} \operatorname{Ext}_{A}^{i-s-1}(L, A)=s+1 .
$$

Proof. By Lemma 1.4(3) we may assume that $L$ is $(s+1)$-pure. By Lemma 1.7, there is a special regular element $x$ such that $\operatorname{Kdim} L / L x=s$. Applying $\operatorname{Ext}_{A}^{i}(-, A)$ to the short exact sequence

$$
0 \rightarrow L \stackrel{x^{n}}{\longrightarrow} L \rightarrow L / L x^{n} \rightarrow 0
$$

we obtain an exact sequence

$$
\rightarrow \operatorname{Ext}_{A}^{i-s-1}(L, A) \rightarrow \operatorname{Ext}_{A}^{i-s-1}(L, A) \rightarrow \operatorname{Ext}_{A}^{i-s}\left(L / L x^{n}, A\right) \rightarrow \operatorname{Ext}_{A}^{i-s}(L, A) \rightarrow .
$$

If $\operatorname{Kdim}_{\operatorname{Ext}}^{i-s-1}(L, A)<s+1$, we may pick $x$ such that $x \operatorname{Ext}_{A}^{i-s-1}(L, A)=0$. Thus we have an exact sequence of modules of $\operatorname{Kdim} \leq s$,

$$
0 \rightarrow \operatorname{Ext}_{A}^{i-s-1}(L, A) \rightarrow \operatorname{Ext}_{A}^{i-s}\left(L / L x^{n}, A\right) \rightarrow \tau_{s} \rightarrow,
$$

where $\tau_{s}=\tau_{s}\left(\operatorname{Ext}_{A}^{i-s}(L, A)\right)$. This shows that the $s$-rank of $\operatorname{Ext}_{A}^{i-s}\left(L / L x^{n}, A\right)$ is bounded for all $n$. By Hypothesis 2.2(2s) the $s$-rank of $\operatorname{Ext}_{A}^{i-s}(L / L x, A)$ is nonzero. Applying Hypothesis 2.2(1s) to the short exact sequence

$$
0 \rightarrow L x / L x^{n} \rightarrow L / L x^{n} \rightarrow L / L x \rightarrow 0,
$$

we see that the $s$-rank of $\operatorname{Ext}_{A}^{i-s}\left(L / L x^{n}, A\right)$ is equal to $n$ times the $s$-rank of $\operatorname{Ext}_{A}^{i-s}(L / L x, A)$. This yields a contradiction, and therefore $\operatorname{Kdim}_{\operatorname{Ext}_{A}^{i-s-1}(L, A)}$ $=s+1$.

Theorem 2.9. Assume Hypothesis 2.1. Then Hypothesis 2.2(1s,2s) holds for all $s$. 
Proof. When $s=0$ the assertion is clear. By induction and Lemma 2.7, Hypothesis $2.2(1 \mathrm{~s})$ holds for all $s$.

Suppose now Hypothesis 2.2(2s) holds. We prove Hypothesis $2.2(2(\mathrm{~s}+1))$ by induction on the $(s+1)$-rank of the noetherian $A$-B-bimodule $M$. By the first paragraph, Hypothesis 2.2(1w) holds for all $w$. By Lemma 1.4(3), it suffices to show that $\operatorname{Kdim~}_{\operatorname{Ext}}^{i-s-1}\left(M / \tau_{s}(M), A\right)=s+1$. So we may assume $M$ is $(s+1)$ pure. If the $(s+1)$-rank of $M$ is 1 , then $M$ is critical and r. $\operatorname{ann}_{B}(M)$ is prime. Replacing $B$ by $B / \mathrm{r}$. $\operatorname{ann}_{B}(M)$, we may assume $B$ is prime. By Lemma 2.8, $\mathrm{Kdim}_{\operatorname{Ext}}^{i-s-1}(M, A)=s+1$ for all $i \in \Phi$, and Hypothesis 2.2(2(s+1)) holds for this special case. Since $\operatorname{Ext}_{A}^{i-s-1}(-, A)$ is exact [Hypothesis 2.2(1(s+1))], induction on the $(s+1)$-rank of $M$ implies that Hypothesis $2.2(2(\mathrm{~s}+1))$ holds for all $M$.

The induction on $s$ completes the proof.

Corollary 2.10. Suppose Hypothesis 2.1 holds.

(1) $\Phi$ is non-empty and consists of a single element $\{d\}$.

(2) $\operatorname{id}{ }_{A} A=d=\operatorname{Kdim} A<\infty$.

Proof. By Theorem 2.9, Hypothesis 2.2(1s,2s) holds for all $s$.

(1) If $\Phi$ is empty, by Lemma 2.3(4), $\operatorname{Hom}_{A}(A, A)=0$. This is impossible. Let $d^{\prime}$ be any element in $\Phi$. Let $M$ be a noetherian $A$-B-bimodule with $\operatorname{Kdim} M=s$. So by Hypothesis 2.2(2s), $\operatorname{Ext}_{A}^{d^{\prime}-s}(M, A) \neq 0$. Since $\operatorname{Ext}^{i}(-, A)=0$ for all $i<0$, it follows that $d^{\prime}-s \geq 0$. This means that $s$ is bounded by $d^{\prime}$, and hence every noetherian $A$ - $B$-bimodule of finite Krull dimension has Krull dimension no more than $d^{\prime}$. It follows from the inductive definition of the Krull dimension that every noetherian $A$ - $B$-bimodule has Krull dimension no more than $d^{\prime}$. Since $A$ itself is a noetherian $A$-A-bimodule (where $B=A$ ), we get $d:=\operatorname{Kdim} A \leq d^{\prime}$. Now Hypothesis $2.2(2 \mathrm{~s})$ implies that

$$
\Phi \subseteq\left\{i \mid \operatorname{Kdim}_{\operatorname{Ext}}^{i-d}(A, A)=d\right\} .
$$

Since $\operatorname{Ext}_{A}^{i}(A, A)=0$ for all $i \neq 0, \Phi=\{d\}$. $d$.

(2) Lemma 2.3(4), Hypothesis 2.1, and the fact that $\Phi=\{d\}$ imply that id ${ }_{A} A=$

If $A$ is not noetherian, then $\Phi$ in Hypothesis 2.1 could be empty, as the next example shows.

Example 2.11. Let $A=\mathbb{C}\left[x_{1}, x_{2}, x_{3}, \cdots\right]$ be the commutative polynomial ring of countably many variables over the field of complex numbers. Then $A$ is a Hopf algebra, and every simple $A$-module is 1 -dimensional over $\mathbb{C}$. It is not noetherian. One can verify that $\operatorname{Ext}_{A}^{i}(S, A)=0$ for all $i$ and all simple $A$-modules $S$. Thus Hypothesis 2.1 holds when $\Phi$ is empty.

\section{The MAIN RESUlts}

We prove Theorems 0.1 and 0.2 in this section. Recall that an algebra is Gorenstein if it has finite left and right injective dimension over itself. There are two stronger variants of the Gorenstein property for noncommutative rings (see the discussions in [Le]). Here is the first one.

Definition 3.1. Let $A$ be a noetherian algebra. We say $A$ is left $A S$-Gorenstein (where AS stands for Artin and Schelter) if 
(1) ${ }_{A} A$ has finite injective dimension, say $d$, and

(2) for every simple $A$-module $S, \operatorname{Ext}_{A}^{i}(S, A)=0$ for all $i \neq d$ and $\operatorname{Ext}^{d}(S, A)$ is a simple $A^{\circ}$-module.

Right AS-Gorensteinness is defined similarly. We say $A$ is $A S$-Gorenstein if it is both left and right AS-Gorenstein.

Proposition 3.2. Let $A$ be a noetherian PI algebra. Then the following are equivalent.

(1) A is AS-Gorenstein.

(2) $A$ is left $A S$-Gorenstein.

(3) A is right $A S$-Gorenstein.

(4) Hypothesis 2.1 holds.

We need a slightly more general version of the double-Ext spectral sequence. This was first proved by Ischebeck [Is, 1.8]; so it is sometimes called Ischebeck's spectral sequence.

Lemma 3.3. Let $A$ and $B$ be algebras. Let $M$ be an $A^{\circ}$-module and $N$ be an $A$-B-bimodule. Suppose that $M_{A}$ has a resolution by finitely generated projectives, and that ${ }_{A} N$ has finite injective dimension. Then there is a convergent spectral sequence of $B^{\circ}$-modules

$$
E_{2}^{p, q}:=\operatorname{Ext}_{A}^{p}\left(\operatorname{Ext}_{A^{\circ}}^{-q}(M, A), N\right) \Rightarrow \operatorname{Tor}_{-p-q}^{A}(M, N) .
$$

Proof of Proposition 3.2. (1) $\Rightarrow(2)$ and (2) $\Rightarrow$ (4) are easy. (2) and (3) are symmetric. So it remains to show $(4) \Rightarrow(1)$.

Assume Hypothesis 2.1 holds. By Corollary 2.10, $A A$ has finite injective dimension, say $d$, and $\Phi=\{d\}$. By Hypothesis 2.1, we see that if $S$ is an $A$-module of finite length, then $\operatorname{Ext}^{i}(S, A)=0$ for all $i \neq d$. By Lemma 1.4(2), $\operatorname{Ext}_{A}^{d}(S, A)$ is an $A^{\circ}$-module of finite length.

The Ischebeck spectral sequence in Lemma 3.3 doesn't require that $A_{A}$ has finite injective dimension. Set $N=A$ and let $M$ be a nonzero finite length $A^{\circ}$ module in Lemma 3.3. Since every $\operatorname{Ext}_{A^{\circ}}^{i}(M, A)$ is an $A$-module of finite length, $\operatorname{Ext}_{A}^{p}\left(\operatorname{Ext}_{A^{\circ}}^{i}(M, A), A\right)=0$ for all $p \neq d$. Hence the spectral sequence collapses to

$$
\left.\operatorname{Ext}_{A}^{d}\left(\operatorname{Ext}_{A^{\circ}}^{q}(M, A), A\right)\right) \cong \operatorname{Tor}_{-d+q}^{A}(M, A)= \begin{cases}M & \text { if } q=d, \\ 0 & \text { if } q \neq d .\end{cases}
$$

Thus Hypothesis 2.1 (when $\Phi=\{d\}$ ) implies that $\operatorname{Ext}_{A^{\circ}}^{d}(M, A) \neq 0$ and that $\operatorname{Ext}_{A^{\circ}}^{q}(M, A)=0$ for all $q \neq d$. The long exact sequence shows that $\operatorname{Ext}_{A^{\circ}}^{q}(-, A)$ is exact on finite length $A^{\circ}$-modules for all $q$. This means that the right-handed version of Hypothesis 2.1 holds. By the right-handed version of Corollary 2.10, id $A_{A}=d$.

By Ischebeck's spectral sequence (when $N=A$ ), we see that the functors $\operatorname{Ext}_{A}^{d}(-, A)$ and $\operatorname{Ext}_{A^{\circ}}^{d}(-, A)$ induce a duality between the category of finite length $A$-modules and that of $A^{\circ}$-modules. In particular, if $S$ is a simple $A$-module, $\operatorname{Ext}_{A}^{d}(S, A)$ is a simple $A^{\circ}$-module. This statement holds when $A$ and $A^{\circ}$ are exchanged. Therefore $A$ is left and right AS-Gorenstein.

We now come to Hopf algebras. We refer to Mon for basic definitions and properties about general Hopf algebras, and to $[\mathrm{Br}$, $\mathrm{BG}$ ] for noetherian PI Hopf algebras. Let $H$ be a Hopf algebra over $k$ and let $k$ also denote the trivial $H$-module 
$H / \epsilon^{-1}(0)$, where $\epsilon: H \rightarrow k$ is the counit. The following lemma is [BG 1.11] and WZ1 4.8]. The proof of [WZ1, 4.8] was given for one $n$, but works for all $n$. Recall that an $H$-module means a left $H$-module.

Lemma 3.4. Let $H$ be a Hopf algebra. Let $n$ be an integer.

(1) The following conditions are equivalent:

(a) $\operatorname{Ext}_{H}^{n}\left({ }_{H} k, H\right) \neq 0$.

(b) $\operatorname{Ext}_{H}^{n}(V, H) \neq 0$ for some finite dimensional $H$-module $V$.

(c) $\operatorname{Ext}_{H}^{n}(V, H) \neq 0$ for all nonzero finite dimensional $H$-modules $V$.

(2) $\operatorname{Ext}_{H}^{n}(-, H)$ is exact on finite dimensional $H$-modules.

Lemma 3.4 is basically saying that Hypothesis 2.1 holds for $H$ if all simple $H$ modules are finite dimensional. We now prove Theorem 0.1.

Theorem 3.5. Let $H$ be a noetherian PI Hopf algebra over $k$ such that every simple $H$-module is finite dimensional over $k$. Then $H$ is AS-Gorenstein.

Proof. By assumption, every simple $H$-module is finite dimensional. Let $\Phi$ be the set of integers $n$ such that $\operatorname{Ext}_{H}^{n}(k, H) \neq 0$. By Lemma 3.4, Hypothesis 2.1 holds for $H$. By Proposition 3.2, $H$ is left and right AS-Gorenstein.

Proof of Theorem 0.1. When $H$ is affine, every simple $H$-module is finite dimensional [MR, 13.10.3(i)]. The assertion follows from Theorem 3.5.

Theorem 0.2 is known for all AS-Gorenstein noetherian PI algebras. Next we recall the second stronger version of the Gorenstein property.

Definition 3.6. We say a noetherian ring $A$ is Auslander-Gorenstein if

(1) $A$ is Gorenstein, i.e., $A$ has finite left and right injective dimension,

(2) for every noetherian $A$-module $M$ and every $A^{\circ}$-submodule $N \subset \operatorname{Ext}_{A}^{j}(M$, $A)$, one has $\operatorname{Ext}_{A^{\circ}}^{i}(N, A)=0$ for all $i<j$, and

(3) part (2) holds when $A$ and $A^{\circ}$ are exchanged.

The grade (or the $j$-number) of $M$ is defined to be

$$
j(M)=\min \left\{i \mid \operatorname{Ext}_{A}^{i}(M, A) \neq 0\right\} .
$$

The same is defined for $A^{\circ}$-modules. Let $\delta$ be a dimension function. We say $A$ is $\delta$-Macaulay if

$$
j(M)+\delta M=\delta A
$$

for all noetherian $A$-modules (and $A^{\circ}$-modules) $M$. In this paper $\delta$ is either Kdim or GKdim. When $\delta=$ GKdim, GKdim-Macaulay is also called Cohen-Macaulay.

A version of depth is defined for non-local algebras in WZ1 p. 521]. Let $A$ be a noetherian ring and let $M$ be a noetherian $A$-module. The depth of $M$ is defined to be

$$
\operatorname{depth} M=\min \left\{i \mid \operatorname{Ext}_{A}^{i}(S, M) \neq 0 \text { for some simple } A \text {-module } S\right\} .
$$

We refer to [WZ1] for the Auslander-Buchsbaum formula, Bass's theorem, and the no-holes theorem for AS-Gorenstein rings. The catenary property is defined in MR. 13.10.13]. A good reference for the catenarity of an Auslander-Gorenstein algebra is [GL].

The following is a collection of some known results. 
Proposition 3.7. Let $A$ be an AS-Gorenstein noetherian PI algebra of injective dimension $d$.

(1) A is Auslander-Gorenstein and Kdim-Macaulay.

(2) A has a quasi-Frobenius artinian ring of fractions.

(3) For every minimal prime $\mathfrak{p}$ of $A, \operatorname{Kdim} A / \mathfrak{p}=d$.

(4) A has a resolution of $A$-bimodules

$$
0 \rightarrow A \rightarrow I^{-d} \rightarrow I^{-d+1} \rightarrow \cdots \rightarrow I^{0} \rightarrow 0
$$

such that when restricted to the left-hand side (respectively, the right-hand side), (3.7.1) is a minimal injective resolution of ${ }_{A} A$ (respectively, $A_{A}$ ), and each $I^{-i}$ is pure of $\operatorname{Kdim} i$ on the left and the right respectively.

(5) If $M$ is a noetherian A-module of finite projective dimension, then $\operatorname{pd} M+$ $\operatorname{depth} M=d$.

(6) If $M$ is a noetherian A-module of finite injective dimension, then id $M=d$.

(7) A is catenary.

Proof. (1) is an ungraded analogue of [SZ, 3.10], and (2,3) follow from [AjSZ, 6.1].

(4) By [YZ2, 4.10], $A$ has a residual complex. By the definition of residual complex [YZ2, 4.3], the resolution has the required properties.

$(5,6)$ are [WZ1, 0.1], and (7) follows from [GL, 1.6] or [YZ1, 2.23].

Corollary 3.8. Let $H$ be a noetherian PI Hopf algebra over $k$ such that every simple $H$-module is finite dimensional. Then $H$ is AS-Gorenstein, and the assertions in Proposition 3.7 hold.

Proof. This follows from Theorem 3.5 and Proposition 3.7.

Proof of Theorem 0.2. By Lemma 1.2(3), Kdim = GKdim when $H$ is affine. Now Theorem 0.2 follows from Corollary 3.8 or Proposition 3.7.

Remark 3.9. We do not assume that a noetherian PI Hopf algebra has a bijective antipode. It is unknown whether every noetherian affine PI Hopf algebra has a bijective antipode.

\section{Projectivity over subalgebras}

We prove Theorem 0.3 in this section. The key property we use is the ASGorenstein property of Hopf algebras $H$ and $H^{\prime}$. First we recall a standard spectral sequence for change of rings (see [Rot, 11.65]).

Lemma 4.1. Let $A \rightarrow B$ be an algebra homomorphism. Let $M$ be an $A$-module and $N$ be a $B$-module. Then there is a convergent spectral sequence

$$
\operatorname{Ext}_{B}^{p}\left(\operatorname{Tor}_{q}^{A}(B, M), N\right) \Rightarrow \operatorname{Ext}_{A}^{p+q}(M, N) .
$$

Lemma 4.2. Let $A$ be a noetherian Gorenstein algebra and let $M$ be a noetherian $A$-module. Then $\operatorname{pd} M<\infty$ if and only if id $M<\infty$.

Proof. Here we need a little bit about complexes and derived functors. Since $A$ has finite left and right injective dimension, $A$ is a dualizing complex over $A$ in the sense of [Ye] (or see [YZ1 1.1]). Let $F$ be the derived functor $\operatorname{RHom}_{A}(-, A)$ and $F^{\circ}$ be the derived functor $\operatorname{RHom}_{A^{\circ}}(-, A)$.

We prove that the assertion holds for bounded complexes $M$ with noetherian cohomologies. First assume pd $M<\infty$. Then $M$ has a bounded resolution by 
noetherian projectives. Since $A$ has finite injective dimension, each noetherian projective has finite injective dimension. Hence $M$ has finite injective dimension. Conversely, if id $M<\infty$, then by [WZ1, 2.1] the bounded complex $F(M)_{A}$ has finite projective dimension. By the above argument, $F(M)_{A}$ has finite injective dimension. By [WZ1, 2.1] again, $M \cong F^{\circ} F(M)$ has finite projective dimension.

Proposition 4.3. Let $A$ and $B$ be noetherian PI AS-Gorenstein algebras of the same injective dimension and let $A \rightarrow B$ be an algebra homomorphism such that $B_{A}$ and ${ }_{A} B$ are noetherian. Then the following are equivalent:

(1) id $B_{A}<\infty$.

(2) $\operatorname{id}_{A} B<\infty$.

(3) $\operatorname{pd} B_{A}<\infty$.

(4) $\operatorname{pd}_{A} B<\infty$.

(5) $B_{A}$ is projective.

(6) ${ }_{A} B$ is projective.

As a consequence, if $A$ has finite global dimension, then $B_{A}$ and ${ }_{A} B$ are projective.

Proof. Let $d=\operatorname{id} B=\operatorname{id} A$.

Let $S$ be a simple $A$-module. Then $\operatorname{Tor}_{q}^{A}(B, S)$ is a $B$-module of finite length. By the AS-Gorenstein property of $B, \operatorname{Ext}_{B}^{p}\left(\operatorname{Tor}_{q}^{A}(B, S), B\right)=0$ for all $p \neq d$. Let $M=S$ and $N=B$ in (4.1.1). Then (4.1.1) becomes

$$
\operatorname{Ext}_{B}^{d}\left(\operatorname{Tor}_{q}^{A}(B, S), B\right) \cong \operatorname{Ext}_{A}^{d+q}(S, B)
$$

for all $q$.

$(2) \Rightarrow(5)$ If id ${ }_{A} B<\infty$, by Bass's theorem in WZ1 0.1(2)], $\operatorname{id}_{A} B=d$. Then $\operatorname{Ext}_{A}^{d+q}(S, B)=0$ for $q>0$. By (4.3.1) and the AS-Gorenstein property of $B$, $\operatorname{Tor}_{q}^{A}(B, S)=0$ for all $q>0$ and for all simple $A$-modules $S$. By Lemma 2.3(5), $\operatorname{pd} B_{A}=0$.

$(1) \Rightarrow(6)$ is similar to $(2) \Rightarrow(5)$.

$(5) \Rightarrow(3)$ and $(6) \Rightarrow(4)$ are trivial. By Lemma $4.2,(1)$ and (3) are equivalent and (2) and (4) are equivalent. Therefore these are all equivalent.

If $A$ has finite global dimension, then (3) and (4) hold. Therefore (5) and (6) hold.

Immediately Proposition 4.3 holds for Hopf algebras in Theorem 3.5, and in particular, we have Theorem 0.3 .

Proof of Theorem 0.3. By Theorem 0.2, $H$ and $H^{\prime}$ are AS-Gorenstein. Hence the assertion follows from Proposition 4.3.

Corollary 4.4. Let $H$ be a Hopf algebra that is a finitely generated module over its affine center. Then there is a central subring $C \subset H$ such that $C \cong k\left[z_{1}, \cdots, z_{d}\right]$ and $H$ is a finitely generated free $C$-module.

Proof. Let $Z$ be the center of $H$, which is an affine commutative ring. By Noether normalization, there is a subring $C \subset Z$ such that $C \cong k\left[z_{1}, \cdots, z_{d}\right]$ and $Z_{C}$ is finitely generated. Here $d=\operatorname{GKdim} Z$, which is equal to GKdim $H$. Since $C$ is another Hopf algebra and regular, by Theorem $0.3, H_{C}$ is projective. Since $C$ is a polynomial ring, projective $C$-modules are free. 
Remark 4.5. There are noetherian commutative Hopf algebras $H \subset H^{\prime}$ such that $H_{H}^{\prime}$ is finitely generated but not free (see [OS] and [Mon, 3.5.2]). By a result of Takeuchi [Ta1, if $H$ is a sub-Hopf algebra of a commutative (or cocommutative) Hopf algebra $H^{\prime}$, then $H_{H}^{\prime}$ is always faithfully flat.

\section{EXTENDED NOETHERIAN CASE}

In this section we study some noetherian PI Hopf algebras that are possibly not affine. If every noetherian PI Hopf algebra is affine, then this section is redundant. Molnar proved that every noetherian commutative Hopf algebra over a field is affine Mol]. Molnar's proof was based on two facts about commutative Hopf algebras: (a) the antipode has finite order (the order is 2 in this case) and (b) there is a bijection between Hopf subalgebras and Hopf ideals. Neither of these has been established for noetherian PI Hopf algebras. At this point we could not solve the following question.

Question 5.1. Is every noetherian (PI) Hopf algebra affine?

We can extend Brown's question to the non-affine case.

Question 5.2. Is every noetherian PI Hopf algebra Gorenstein?

We don't have an answer to Question 5.2 either. A $k$-algebra $A$ is called extended noetherian if for every field extension $K \supset k, A \otimes_{k} K$ is noetherian. We show that some versions of Theorems 0.1, 0.2 and 0.3 hold for extended noetherian PI Hopf algebras. If the answer to Question 5.1 is negative, then it is worth presenting this section. Another reason why we present this section is that some ideas here apply to general extended noetherian rings. There are many extended noetherian rings that are not affine (e.g., $A=k(x)$ ); however, we don't know of any extended noetherian PI Hopf algebra that is not affine.

Let $K$ be a field extension of $k$. We write $A_{K}$ for the algebra $A \otimes_{k} K$, and $M_{K}$ for $M \otimes_{k} K$. We also use $\operatorname{Kdim}_{K} M_{K}$ for the Krull dimension of $M_{K}$ as an $A_{K}$-module.

Lemma 5.3. Let $A$ be a noetherian PI k-algebra.

(1) If $A$ is affine, then $A$ is extended noetherian.

(2) If $A$ is extended noetherian, then GKdim $A<\infty$.

(3) If $A$ is extended noetherian, then there is a field extension $K$ such that every simple $A_{K}$-module is finite dimensional.

(4) Let GKdim $A=s<\infty$. Let $K$ be a field extension of $k$ such that (a) $A \otimes_{k} K$ is noetherian and (b) $K \supset k\left(t_{1}, \cdots, t_{s}\right)$. Then $\operatorname{Kdim}_{K} M_{K}=\operatorname{GKdim} M$ for all noetherian A-modules $M$.

Proof. (1) This is a special case of [Sm, Proposition 53]. A proof was also given in ArSZ, 4.4].

(2) By Lemma 1.1(2) we may assume $A$ is prime. Let $Q$ be the ring of fractions of $A$. Then $Q$ is extended noetherian. Let $Z$ be the center of $Q$; then $Z$ is extended noetherian and $Q$ is a finitely generated module over $Z$ [Row 6.1.25]. By a result of Vámos [Va, 11], $Z \otimes_{k} Z$ is noetherian if and only if $Z$ is finitely generated as a field extension. Hence GKdim $Z<\infty$ Row 6.3.41]. This implies that $Q$ and $A$ have finite Gelfand-Kirillov dimension. 
(3) Let $K$ be a field extension of $k$ such that (a) $K$ is algebraically closed and (b) the cardinality of $K$ is bigger than the number of $k$-algebra generators of $A$. Then by [Row 6.1.24 and 6.1.15] every simple $A_{K}$-module is finite dimensional.

(4) Without loss of generality, we may assume that $K=k\left(t_{1}, \cdots, t_{s}\right):=K_{s}$. We use induction on $\operatorname{Kdim} M$. By the reduction in the proof of Lemma 1.4(2) we may assume $M=A / \mathfrak{p}$ for some prime $\mathfrak{p}$. Without loss of generality, we may assume $\mathfrak{p}=0$ and $A$ is prime with an artinian ring of fractions, say $Q$. Let $n$ be GKdim $A$, which is no more than $s$. Since $Q$ is obtained by inverting central elements in $A$ [MR 13.6.5], GKdim $Q=\operatorname{GKdim} A$ [MR, 8.2.13]. Letting $Z$ be the center of $Q$, we see by Noether normalization that $Z$ contains a copy of $K_{n}$. Since $K_{n} \otimes_{k} K_{n}$ has Krull dimension $n$, the Krull dimension of $Q \otimes_{k} K_{n}$ is at least $n$ [MR, 6.6.17]. Therefore $\operatorname{Kdim}_{K} A_{K} \geq \operatorname{Kdim}_{K_{n}} A_{K_{n}} \geq n$ [MR 6.5.3]. By Lemma 1.1(5), $\operatorname{Kdim}_{K} A_{K} \leq \operatorname{GKdim} A \otimes_{k} K=n$ [Row, 6.2.26'].

Lemma 5.4. Let $A$ be a noetherian algebra. Let $M$ and $N$ be noetherian $A$ modules. Then for every $i$ and for every field extension $K \supset k$,

$$
\operatorname{Ext}_{A_{K}}^{i}\left(M_{K}, N_{K}\right) \cong \operatorname{Ext}_{A}^{i}(M, N) \otimes_{k} K .
$$

Proof. This is true for $M=A$. The assertion follows from the fact that $M$ has a resolution by finite free $A$-modules.

Lemma 5.5. Let $A$ be a noetherian algebra and let $M$ be a noetherian A-module. Let $K$ be a field extension such that $A_{K}$ is noetherian.

(1) $\operatorname{pd} M_{K}=\operatorname{pd} M$.

(2) id $M_{K} \geq$ id $M$.

(3) If $A_{K}$ is Gorenstein, so is $A$.

(4) If $A_{K}$ is Auslander-Gorenstein, so is $A$.

(5) $j\left(M_{K}\right)=j(M)$.

(6) If $A_{K}$ is Cohen-Macaulay, so is $A$.

Proof. (1) Since the faithfully flat functor $-\otimes_{k} K$ preserves projective modules, it preserves pd.

(2) follows from Lemma 5.4, and (3) is a special case of (2).

$(4,5)$ Both of these follow from Lemma 5.4 and the definitions.

(6) This follows from (5) and the fact that GKdim is preserved by field extensions Row 6.2.26'].

Theorem 5.6. Let $H$ be an extended noetherian PI Hopf algebra over a field $k$ and let $d$ be the injective dimension of ${ }_{H} H$.

(1) There is a field extension $K$ such that $H_{K}$ is AS-Gorenstein and the conclusions in Proposition 3.7 hold for the Hopf algebra $H_{K}$.

(2) $H$ is Gorenstein, and id $H=$ id $H_{K}$. In particular, $d$ is finite.

(3) GKdim $H=d$.

(4) $H$ is Auslander-Gorenstein and Cohen-Macaulay.

(5) $\operatorname{Kdim} H \leq d$.

(6) $H$ has a quasi-Frobenius artinian ring of fractions.

(7) For every minimal prime $\mathfrak{p}$ of $H$, GKdim $H / \mathfrak{p}=d$.

(8) $H$ has a resolution of $H$-bimodules

$$
0 \rightarrow H \rightarrow I^{-d} \rightarrow I^{-d+1} \rightarrow \cdots \rightarrow I^{0} \rightarrow 0
$$


such that when restricted to the left-hand side (respectively, the right-hand side), (5.6.1) is a minimal injective resolution of ${ }_{H} H$ (respectively, $H_{H}$ ), and each $I^{-i}$ is pure of GKdim $i$ on the left and the right respectively.

(9) If $M$ is a noetherian $H$-module of finite projective dimension, then $\operatorname{pd} M \leq$ $d$.

(10) If $M$ is a noetherian $H$-module of finite injective dimension, then id $M \leq d$.

(11) $H$ is catenary.

Proof. We choose an extension field $K \supset k$ such that the conclusions of Lemma $5.3(3,4)$ hold.

(1) This follows from Lemma 5.3(3) and Corollary 3.8.

(2) Let $n=\mathrm{id} H_{K}$. Since $H_{K}$ is AS-Gorenstein (see (1)), it follows that $n$ is finite and $\operatorname{Ext}_{H_{K}}^{n}\left(K, H_{K}\right) \neq 0$. By Lemma 5.4, $\operatorname{Ext}_{H}^{n}(k, H) \neq 0$. Thus id $H \geq n$. By Lemma 5.5(2), id $H \leq$ id $H_{K}=n$. Therefore $n=d$.

(3) By Proposition 3.7(3) for $H_{K}$,

$$
\operatorname{Kdim}_{K} H_{K}=\max _{\mathfrak{p}}\left\{\operatorname{Kdim} H_{K} / \mathfrak{p}\right\}=d,
$$

where $\mathfrak{p}$ runs over all minimal prime ideals of $H_{K}$. By the choice of $K$, we have GKdim $H=\operatorname{Kdim}_{K} H_{K}=d$.

(4) This follows from (1) and Lemma 5.5(4,6).

(5) This follows from (3) and Lemma 1.1(5).

$(6,7)$ These follow from (4) and AjSZ, 6.1].

(8) This follows from (4) and YZ2, 4.10].

(9) By (1) and Proposition 3.7(5), pd $M_{K}=d-\operatorname{depth} M_{K} \leq d$. The assertion follows from Lemma 5.5(1).

(10) Since $H$ is Gorenstein, by Lemma 4.2, pd $M<\infty$. By Lemma 5.5(1), pd $M_{K}<\infty$. By Lemma 4.2 again, id $M_{K}<\infty$. By Proposition 3.7(6), id $M_{K}=d$. By Lemma 5.5(2), id $M \leq$ id $M_{K}$.

(11) This follows from (4) and [GL, 1.6] or [YZ1, 2.23].

Corollary 5.7. If $H$ is an extended noetherian PI Hopf algebra and every simple $H$-module is finite dimensional, then $\operatorname{Kdim} M=\mathrm{GKdim} M$ for all noetherian $H$ modules $M$.

Proof. By Theorem 5.6(4), $H$ is Cohen-Macaulay. By Corollary 3.8, $H$ is KdimMacaulay. Hence Kdim = GKdim .

Corollary 5.8. Let $H$ and $H^{\prime}$ be extended noetherian PI Hopf algebras of the same injective dimension. Suppose that there is an algebra homomorphism $H \rightarrow H^{\prime}$ such that $H^{\prime}$ is a noetherian $H$-module on both sides. If either $\mathrm{id}_{H} H^{\prime}<\infty$ or $\operatorname{pd}_{H} H^{\prime}<\infty$, then $H_{H}^{\prime}$ and $H_{H} H^{\prime}$ are projective.

Proof. By Lemma 5.3(3), there is a field extension $K$ such that the simple modules over $A:=H \otimes_{k} K$ and over $B:=H^{\prime} \otimes_{k} K$ are finite dimensional over $K$. By Theorem 5.6(1), $A$ and $B$ are AS-Gorenstein. Hence the algebra map $A \rightarrow B$ satisfies the hypotheses in Proposition 4.3. If either id ${ }_{H} H^{\prime}<\infty$ or $\operatorname{pd}_{H} H^{\prime}<\infty$, then, by Lemmas 4.2 and 5.5(1), id ${ }_{A} B<\infty$ and $\mathrm{pd}{ }_{A} B<\infty$. By Proposition 4.3, $B$ is projective over $A$ on both sides. By Lemma 5.5(1), $H^{\prime}$ is projective over $H$ on both sides.

Remark 5.9. We don't know of any noetherian Hopf algebra that is not Gorenstein. So it is natural to extend Brown's question [Br, Question A] to the non-PI case: 
is every noetherian Hopf algebra Gorenstein? We have shown in WZ2 that this is true for graded rings with balanced dualizing complexes: Let $H$ be a noetherian locally finite $\mathbb{N}$-graded Hopf algebra. If $H$ has a balanced dualizing complex, then it has finite left and right injective dimension.

\section{ACKNOWLEDGMENTS}

We thank Ken Brown and Paul Smith for useful conversations on the subject and for their careful reading of an earlier version of the manuscript. We thank the referee for her/his careful reading of the manuscript and valuable comments.

\section{REFERENCES}

[AjSZ] K. Ajitabh, S. P. Smith and J. J. Zhang, Auslander-Gorenstein rings, Comm. Algebra, 26 1998, 2159-2180. MR 99g:16010

[ArSZ] M. Artin, L. W. Small and J. J. Zhang, Generic flatness for strongly Noetherian algebras, J. Algebra 221 (1999), 579-610. MR 2001a:16006

[Br] K. A. Brown, Representation theory of Noetherian Hopf algebras satisfying a polynomial identity, Trends in the representation theory of finite-dimensional algebras (Seattle, WA, 1997), 49-79, Contemp. Math., 229, American Mathematical Society, Providence, RI, 1998. MR 99m:16056

[BG] K. A. Brown and K. R. Goodearl, Homological aspects of Noetherian PI Hopf algebras and irreducible modules of maximal dimension, J. Algebra 198 (1997), 240-265. MR 99c:16036

[DG] M. Demazure and P. Gabriel, "Introduction to algebraic geometry and algebraic groups", Translated from the French by J. Bell, North-Holland Mathematics Studies, 39. NorthHolland Publishing Co., Amsterdam-New York, 1980. MR 82e:14001

[GL] K. R. Goodearl and T. H. Lenagan, Catenarity in quantum algebras, J. Pure Appl. Algebra, 111 (1996), 123-142. MR 97e:16054

[GW] K. R. Goodearl and R. B. Warfield, Jr., "An Introduction to Noncommutative Noetherian Rings," London Math. Soc. Student Texts, Vol. 16, Cambridge Univ. Press, Cambridge, 1989. MR 91c:16001

[Is] F. Ischebeck, Eine Dualität zwischen den Funktoren Ext und Tor (German), J. Algebra 11 (1969), 510-531. MR 38:5894

[KL] G. R. Krause and T. H. Lenagan, "Growth of algebras and Gelfand-Kirillov dimension", Revised edition, Graduate Studies in Mathematics, 22. American Mathematical Society, Providence, RI, 2000. MR 2000j:16035

[LR1] R. G. Larson and D. E. Radford, Finite-dimensional cosemisimple Hopf algebras in characteristic 0 are semisimple, J. Algebra 117 (1988), no. 2, 267-289. MR 89k:16016

[LR2] R. G. Larson and D. E. Radford, Semisimple cosemisimple Hopf algebras, Amer. J. Math. 110 (1988), no. 1, 187-195. MR 89a:16011

[LS] R. G. Larson and M. Sweedler, An associative orthogonal bilinear form for Hopf algebras, Amer. J. Math. 91 (1969) 75-94. MR 39:1523

[Le] T. Levasseur, Some properties of noncommutative regular graded rings, Glasgow Math. J. 34 (1992), 277-300. MR 93k:16045

[MR] J. C. McConnell and J. C. Robson, "Noncommutative Noetherian Rings," Wiley, Chichester, 1987. MR 89j:16023

[Mol] R. K. Molnar, A commutative Noetherian Hopf algebra over a field is finitely generated. Proc. Amer. Math. Soc. 51 (1975), 501-502. MR 51:12915

[Mon] S. Montgomery, Hopf algebras and their actions on rings. CBMS Regional Conference Series in Mathematics, 82, American Mathematical Society, Providence, RI, 1993. MR 94i:16019

[NR] W. D. Nichols and M. B. Richmond, Freeness of infinite-dimensional Hopf algebras. Comm. Algebra 20 (1992), no. 5, 1489-1492. MR 93d:16054

[NZ] W. D. Nichols and M. B. Zoeller, A Hopf algebra freeness theorem. Amer. J. Math. 111 (1989), no. 2, 381-385. MR 90c:16008 
[OS] U. Oberst and H.-J. Schneider, Untergruppen formeller Gruppen von endlichem Index. (German) J. Algebra 31 (1974), 10-44. MR 50:13057

[Po] N. Popescu, Abelian categories with applications to rings and modules. London Mathematical Society Monographs, No. 3. Academic Press, London-New York, 1973. MR 49:5130

[Ra] D. E. Radford, Freeness (projectivity) criteria for Hopf algebras over Hopf subalgebras, J. Pure Appl. Algebra 11 (1977/78), no. 1-3, 15-28. MR 57:16344

[Rot] J. J. Rotman, "An introduction to homological algebra", Pure and Applied Mathematics, 85. Academic Press, Inc. New York-London, 1979. MR 80k:18001

[Row] L. H. Rowen, "Ring theory", Student edition. Academic Press, Inc., Boston, MA, 1991. MR 94e:16001]

[Sm] L.W. Small, Rings satisfying a polynomial identity, Lecture Notes, Universität Essen (1980). MR 82j:16028

[SZ] J. T. Stafford and J. J. Zhang, Homological properties of (graded) noetherian PI rings, J. Algebra 168 (1994), 988-1026. MR 95h:16030

[Ta1] M. Takeuchi, A correspondence between Hopf ideals and sub-Hopf algebras, Manuscripta Math. 7 (1972), 251-270. MR 48:328

[Ta2] M. Takeuchi, Relative Hopf modules - equivalences and freeness criteria, J. Algebra 60 (1979), no. 2, 452-471. MR 82m:16006

[Va] P. Vámos, On the minimal prime ideal of a tensor product of two fields, Math. Proc. Cambridge Philos. Soc. 84 (1978), no. 1, 25-35. MR 80j:12016

[WZ1] Q.-S. Wu and J. J. Zhang, Homological identities for noncommutative rings, J. Algebra, 242 (2001), 516-535.

[WZ2] Q.-S. Wu and J. J. Zhang, Gorenstein Property of Hopf Graded Algebras, Glasgow Math. J., (to appear).

[WZ3] Q.-S. Wu and J. J. Zhang, Regularity of Involutory PI Hopf Algebras, J. Algebra, (to appear).

[Ye] A. Yekutieli, Dualizing complexes over noncommutative graded algebras, J. Algebra 153 (1992), 41-84. MR 94a:16077

[YZ1] A. Yekutieli and J. J. Zhang, Rings with Auslander dualizing complexes, J. Algebra 213 (1999), 1-51. MR 2000f:16012

[YZ2] A. Yekutieli and J. J. Zhang, Residue complexes over noncommutative rings, J. Algebra, to appear. ArXiv: math.RA/0103075.

Institute of Mathematics, Fudan University, Shanghai, 200433, China

E-mail address: qswu@fudan.edu.cn

Department of Mathematics, Box 354350, University of Washington, Seattle, WashINGTON 98195

E-mail address: zhang@math.washington.edu 